\title{
Queueing Analysis of Opportunistic Scheduling with Spatially Correlated Channels
}

\author{
Ekaterina Evdokimova ${ }^{\mathrm{a}}$, Koen De Turck ${ }^{\mathrm{b}}$, Sabine Wittevrongel ${ }^{\mathrm{a}}$, Dieter Fiems ${ }^{\mathrm{a}, *}$ \\ ${ }^{a}$ Ghent university, Department of Telecommunication and Information Processing, Sint-Pietersnieuwstraat 41, 9000 Ghent, \\ Belgium \\ ${ }^{b}$ Laboratoire des Signaux et Systèmes (UMR8506), CentraleSupélec, 3, Rue Joliot-Curie, 91192 Gif-sur-Yvette, France
}

\begin{abstract}
Exploiting the differences in supported transmission rates between mobile users, opportunistic scheduling promises a substantial increase of the aggregate throughput of wireless networks. In this paper, we present a Markov model to study the trade-off between fairness and wireless efficiency of opportunistic scheduling at an access point which serves multiple mobile users. The Markov process at hand tracks the queues of outstanding packets for the different mobile users as well as the states of the wireless channels for these users. Because the size of the state space of the Markov model prevents a direct solution, we develop a numerical analysis technique based on Maclaurin series expansions to solve the system in light traffic and in overload. By numerical examples we illustrate the accuracy of our approach and compare a set of performance metrics of various schedulers. In particular, we study how cross-channel correlation affects the performance of these schedulers.
\end{abstract}

\section{Introduction}

Efficiently allocating networking resources is key for the performance of many multi-user (MU) wireless communication systems. Such allocations aim at optimising performance metrics like network throughput, delay and jitter, while at the same time retaining fairness between the users [1]. In contrast to wireline networks, the transmission rates supported by individual mobile users vary over time and per user. For example, the channel capacity of an individual user depends on its distance to the base station. In addition, users undergo short-term location-dependent fading, the statistics of such short-term variations differing across users [2]. Therefore, scheduling to the users with the best rates at any given time may allow for substantially increasing the aggregate throughput of the wireless network.

Such opportunistic scheduling is a promising cross-layer technique that holds the potential of significantly improving wireless networks' efficiency in the near future. The technique however immediately brings into

\footnotetext{
*Corresponding author

Email addresses: Ekaterina.Evdokimova@UGent.be (Ekaterina Evdokimova), koen.deturck@l2s.centralesupelec.fr (Koen De Turck), Sabine.Wittevrongel@UGent.be (Sabine Wittevrongel), Dieter.Fiems@UGent.be (Dieter Fiems)
} 
light the trade-off between wireless efficiency (i.e., a preference to schedule to the best channel) and fair scheduling (i.e., each user is entitled to a certain amount of network resources) [3]. Since the introduction of opportunistic scheduling in [4], numerous schedulers have been proposed for different instances of wireless networks, such as mobile cellular networks, cognitive radio, WiMAX, MIMO systems; see [5-12] and the references therein.

While holding the promise to increase the throughput, opportunistic scheduling also faces limitations. In order to select where to send to, the scheduler requires accurate channel state information (CSI). Such CSI is reported at the low rate of the feedback channel, and may therefore be outdated at the time the scheduler decides where to send to. In other words, the opportunistic scheduler must always rely on partially known channel state information, the timeliness depending on the feedback delay. The discussion how much feedback is sufficient in order to benefit from the MU diversity is actively investigated [13, 14]. Furthermore, the gain of opportunistic scheduling may suffer from cross-channel correlations between users [15]. Typically the wireless networks exploiting MU diversity achieve the maximum performance in case of independent channels, positive cross-correlation leading to a decrease of the overall transmission capacity [16, 17]. Finally, the scheduling policy itself cannot require excessive computational complexity. While literature often focuses on designing optimal schedulers for particular fairness and quality-of-service (QoS) requirements, such schedulers may require too much computations to be practically implementable. Therefore, heuristic approaches are often preferred in order to simplify the implementation $[18,19]$. Although many schedulers have been proposed, for the next generation of wireless systems there is a demand for new policies that rely on less feedback information, account for the spatial correlations between users and require low computational complexity, while providing nearly optimal scheduling decisions. See for example $[5,8,19-22,55-57]$ for advances in opportunistic scheduling policies.

Along with developments in scheduling strategies, there is also an increasing demand for performance evaluation tools (i.e., instruments for testing, comparing and designing schedulers) for opportunistic schedulers. Only few authors assess the performance of opportunistic schedulers by analytic means, most assessments of schedulers relying on simulations, see e.g. [22-26]. This is not surprising as stochastic models of opportunistic schedulers involve multiple queues. This results in Markov models with a multidimensional state spaces. Even for a limited number of buffers (or mobile nodes) and limited buffer capacities, the state space of the Markov chain is huge which makes direct solution techniques numerically unfeasible. In [27], the authors propose a decomposition method to avoid the state space explosion problem. The approach relies on representing the MU system with $K$ mobile users by a deterministic stochastic Petri-net (DSPN), decomposed into $K$ subnets. Since the subnets are analysed separately, the MU system is represented with far fewer states than the original Markov model, thereby achieving a low computational complexity. This approach however rules out most interactions between the mobile users which is essential for a complete performance study. Indeed, the interaction is key for the scheduler as each allocation decision impacts all 
mobile users. A similar decomposition approach is presented in [28] for cognitive radio spectrum allocation. Here, a queueing model is analysed by matrix-analytic methods. However, the study mainly focuses on the single-queue case with an extrapolation to multiple queues. Finally, [19] studies the formation of time-space batches of packets assigned for simultaneous transmission. In this paper, the authors do not track the number of packets for the different destinations, thereby again avoiding the inherent multidimensionality.

This paper proposes an analytic framework for studying opportunistic schedulers. Specifically, we propose a method for the fast performance evaluation of wireless networks equipped with one access point (AP) serving multiple mobile users under varying transmission conditions. Our approach neither relies on decomposition nor on an extrapolation of the single-queue case, which enables us to accurately study the interactions among the queues. We consider a continuous-time Markovian model with a separate queueing state variable for each mobile node. Assuming Rayleigh fading, our channel model not only accounts for temporal correlation, but also for spatial correlation between the channels. The effects of fading are introduced in the queueing model by a transmission environment variable. The transmission environment is an exogenous continuous-time Markov process with a finite number of states in accordance with [29]. The overall queueing model at hand is a continuous-time Markov process. This means that opportunistic scheduling is modelled as an assignment of transmission rates to queues, thereby (possibly) accounting for both the current number of packets in the queue as well as the channel conditions. The transmission rate being proportional to the chance of a service time completion in an infinitely small interval, such a model still allows for some uncertainty in having a successful transmission or in the estimation of the channel conditions, but largely makes abstraction of the details of scheduling the packets.

The size of the state space of the Markov process at hand makes a direct solution technique computationally unfeasible. For example, a system with 10 mobile nodes and buffer capacity for storing 10 packets per mobile node yields a state space with size exceeding $10^{10}$. To cope with such state spaces, we rely on Maclaurin series expansions of the solution of the Markov process to assess the performance both fast and accurately [31-34]. Depending on the context in which they are introduced, series expansion techniques for Markov chains are referred to as perturbation techniques, the power series algorithm or light-traffic analysis. While the naming is not absolute, perturbation methods are mainly motivated by the assessment of the sensitivity of the performance measures with respect to a system parameter. The case where the perturbation does not preserve the class structure of the non-perturbed chain — the so-called singular perturbations has received much attention in literature [31,35]. The power series algorithm transforms a Markov chain of interest in a set of Markov chains parametrised by a possibly artificial parameter. When the parameter is zero, the chain is not only easily solved, but one can also obtain the series expansion in the parameter. When the parameter is one, one gets the original Markov chain such that the series expansion can be used to approximate the solution of the original Markov chain, provided the radius of convergence of the series expansion exceeds one [36]. Finally, light-traffic analysis often corresponds to a series expansion in the ar- 


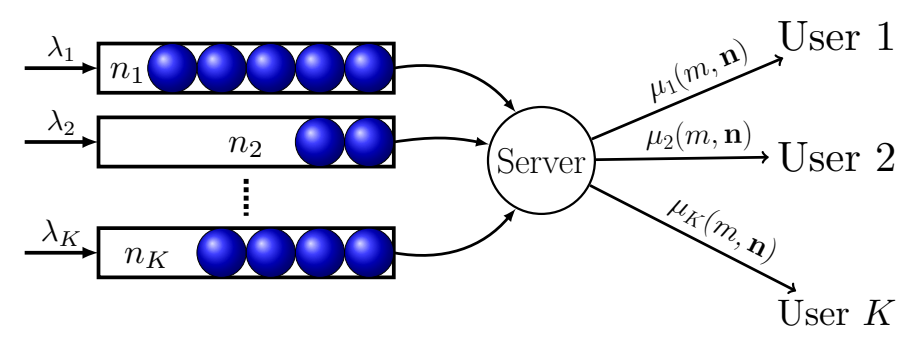

Figure 1: Queueing model for the opportunistic scheduler

rival rate at a queue. For an overview on the technique of series expansions in stochastic systems, we further refer the reader to the surveys in [37] and [38] and the recent book [39]. The present study most closely relates to the numerical series expansion approach of [33] and [34]. In contrast to this work, the present unperturbed chain is not upper-diagonal, but block upper-diagonal. It is shown below, that calculating the terms in the series expansion — in overload as well as under light traffic — is much easier than solving the queueing model for any particular load. The present paper extends our preliminary findings presented in $[40,41]$.

The remainder of the paper is organised as follows. The next section introduces the modelling assumptions and settles the notational conventions. The proposed analysis technique is then outlined in Section 3. Section 4 discusses the Markovian Rayleigh fading channel model. In order to illustrate and assess the proposed performance evaluation method, we consider some numerical examples in section 5 and validate the accuracy of our results by simulation. In particular, we implement several simple schedulers [4, 34, 42, 43] for systems with spatially independent and correlated channels. Finally, conclusions are drawn in Section 6.

\section{Queueing model}

We consider a wireless AP, opportunistically sending packets to multiple mobile nodes. The AP is modelled as a Markovian queueing model with $K$ finite-capacity queues that share a common transmission channel, as depicted in Figure 1. Each queue corresponds to the AP buffer of a particular mobile node. Let $N_{k}(t)$ be the number of packets in the buffer of the $k$ th mobile node at time $t$, let $C_{k}$ be the capacity of this buffer and let $\mathbf{N}(t)=\left[N_{1}(t), \ldots, N_{K}(t)\right]$ be the vector with elements $N_{k}(t)$. Arrivals at the different buffers are modelled by independent Poisson processes; $\lambda_{k}$ denotes the arrival rate at queue $k$. We further assume that the packet sizes in the $k$ th queue are exponentially distributed with rate $\theta_{k}$.

The mobile nodes experience different time-varying channel conditions. To model variations of the channel conditions in both space and time, we introduce an exogenous continuous-time Markov process $M(t)$ that modulates the states of the different wireless transmission channels. We refer to $M(t)$ as the background process. Let the finite set $\mathcal{M}$ be the state space of this Markovian background process, let $M$ denote the cardinality of $\mathcal{M}$, and let $\alpha_{i j}$ denote the transition rate from state $i$ to state $j, i \neq j, i, j \in \mathcal{M}$. 
The corresponding generator matrix is denoted by $A=\left[\alpha_{i j}\right]_{i, j \in \mathcal{M}}$, with $\alpha_{i i} \doteq-\sum_{j \in \mathcal{M} \backslash\{i\}} \alpha_{i j}$ for $i \in \mathcal{M}$. For every background state $m \in \mathcal{M}$, let $\mathbf{g}_{m}=\left[g_{m 1}, \ldots, g_{m K}\right]$ be a vector whose $k$ th element quantifies the channel conditions as experienced by the $k$ th mobile node. Hence, the channel condition vector at time $t$ is $\mathbf{G}(t):=\mathbf{g}_{M(t)}$. Without loss of generality, we assume that $g_{m k} \in[0 \ldots 1]$, where $g_{m k}=1$ corresponds to the best expected channel quality that allows transmission at the highest rate and $g_{m k}=0$ represents the case of poor channel quality when transmission is not feasible.

Given the channel conditions $\mathbf{G}(t)$ and the number of packets in the queues $\mathbf{N}(t)$, the opportunistic scheduler assigns service rates to the different queues. Let $\Psi_{k}(\mathbf{g}, \mathbf{n})$ be the rate assigned to the $k$ th mobile node, assuming channel conditions $\mathbf{G}(t)=\mathbf{g}$ and queueing state $\mathbf{N}(t)=\mathbf{n}$. We do not make any additional assumptions on the scheduling rule. Various specific schedulers are studied in section 5 including MaxRate, MaxWeight, Longest Connected Queue, and some schedulers that relate to the generalised and differentiated processor sharing service disciplines.

In view of the assumptions above, the stochastic process $[\mathbf{N}(t), M(t)]$ is a Markov process. Accounting for the packet size distribution, for queueing state $\mathbf{N}(t)=\mathbf{n}$ and background state $M(t)=m$, packets depart from queue $k$ with rate,

$$
\mu_{k}(\mathbf{n}, m)=\theta_{k} \Psi_{k}\left(\mathbf{g}_{m}, \mathbf{n}\right)
$$

such that the total departure rate in state $(\mathbf{N}(t), M(t))=(\mathbf{n}, m)$ equals,

$$
\mu(\mathbf{n}, m)=\sum_{k=1}^{K} \mu_{k}(\mathbf{n}, m) .
$$

The following notation will simplify the equations below. Let $\mathcal{C}_{k}=\left\{0,1, \ldots, C_{k}\right\}$ be the set of possible states of the $k$ th queue and let $\mathcal{C}=\mathcal{C}_{1} \times \ldots \times \mathcal{C}_{K}$. The state space of the Markovian queueing model is then $\mathcal{C} \times \mathcal{M} ; S$ denotes the size of the state space $\mathcal{C} \times \mathcal{M}$. Also, $\mathbf{c}=\left[C_{1}, \ldots, C_{K}\right]$ corresponds to the case where all buffers are full; $\mathcal{M}_{\mathbf{c}}=\{[\mathbf{c}, j], j \in \mathcal{M}\}$ denotes the corresponding subset of the state space. We define $\mathbf{e}_{k}$ as the row vector of length $K$ with its $k$ th element set to 1 and all other elements equal to zero and define e as the row vector of ones. Finally, we introduce the global arrival rate $\lambda$ and global service rate $\mu$ which allow to simultaneously scale all the arrival rates or all service rates.

Remark 1. At the level of abstraction of the queueing model at hand, we did not specify any technological assumptions on the AP under consideration. The model at hand allows to assess the performance of the buffer behaviour at the AP for wireless systems with opportunistic scheduling like cognitive radio, micro-cell networks, Wi-Fi or WiMAX networks, and for different configurations of MU MIMO with a single AP. In particular, the present study allows for simultaneous transmissions to multiple users, while the modelling assumptions are sufficiently versatile to capture a variety of channel- and buffer-aware policies that base their scheduling decisions on the current state of the system and transmission environment. 


\section{Performance analysis}

Having specified the modelling assumptions, we now present the numerical analysis technique. We first introduce the balance equations of the Markov chain under consideration. Expanding the stationary distribution of the Markov chain around $\mu=0$ and $\lambda=0$, with $\mu$ the global service rate and $\lambda$ the global arrival rate, we derive approximations for the stationary distribution and various performance measures in the light-traffic and the overload regime, respectively.

\subsection{Balance equations}

In view of the modelling assumptions introduced above, the state of the system is described by the vector $(\mathbf{n}, j)$ where the vector $\mathbf{n}=\left[n_{1}, \ldots, n_{K}\right]$ collects the states of the queues $-n_{k}$ denotes the number of packets in the $k$ th queue - and where $j \in \mathcal{M}$ denotes the state of the background process. Moreover, let $\pi(\mathbf{n}, j)=\lim _{t \rightarrow \infty} \mathrm{P}[\mathbf{N}(t)=\mathbf{n}, M(t)=j]$ be the steady-state probability to be in state $(\mathbf{n}, j)$. As the state space of the Markov chain at hand is finite, the steady-state probabilities are well-defined provided the Markov chain has a single ergodic class. This is the case if (i) the background process $M(t)$ is stationary ergodic, (ii) $\lambda_{k}>0$ for all $k \in\{1, \ldots, K\}$ and, (iii) $\sum_{j \in \mathcal{M}} \mu_{k}(\mathbf{n}, j)>0$ for all $\mathbf{n} \in \mathcal{C} \backslash\{\mathbf{0}\}$ and $k \in\{1, \ldots, K\}$. If one relaxes the assumption that queue capacity is finite, conditions for the existence of a stationary solution are not entirely trivial, see e.g. [30] where the stability of a slightly different queueing system (with infinite capacities) is addressed.

As there are neither simultaneous arrivals nor departures, we find the following set of balance equations,

$$
\begin{gathered}
\pi(\mathbf{n}, j)\left(\sum_{k=1}^{K}\left(\lambda_{k} \mathbf{1}_{\left\{n_{k}<C_{k}\right\}}+\mu_{k}(\mathbf{n}, j) \mathbf{1}_{\left\{n_{k}>0\right\}}\right)+\sum_{i \in \mathcal{M} \backslash\{j\}} \alpha_{j i}\right) \\
=\sum_{k=1}^{K} \pi\left(\mathbf{n}+\mathbf{e}_{k}, j\right) \mu_{k}\left(\mathbf{n}+\mathbf{e}_{k}, j\right) \mathbf{1}_{\left\{n_{k}<C_{k}\right\}} \\
+\sum_{k=1}^{K} \pi\left(\mathbf{n}-\mathbf{e}_{k}, j\right) \lambda_{k} \mathbf{1}_{\left\{n_{k}>0\right\}}+\sum_{i \in \mathcal{M} \backslash\{j\}} \pi(\mathbf{n}, i) \alpha_{i j},
\end{gathered}
$$

for $\mathbf{n} \in \mathcal{C}$ and $j \in \mathcal{M}$. Here $\mathbf{1}_{\{\cdot\}}$ is the indicator which evaluates to one if its argument is true and to zero if this is not the case. State transitions correspond to arrivals at and departures from the different queues, or to state transitions of the channel. For ease of notation, we group the stationary probabilities for a given queueing state into vectors $\boldsymbol{\pi}(\mathbf{n})=[\pi(\mathbf{n}, j)]_{j \in \mathcal{M}}$. We can then rewrite the balance equations as follows,

$$
\begin{aligned}
\boldsymbol{\pi}(\mathbf{n})\left(\sum_{k=1}^{K}\left(\lambda_{k} \mathbf{1}_{\left\{n_{k}<C_{k}\right\}} I_{M}+M_{k}(\mathbf{n}) \mathbf{1}_{\left\{n_{k}>0\right\}}\right)-A\right) \\
=\sum_{k=1}^{K} \boldsymbol{\pi}\left(\mathbf{n}+\mathbf{e}_{k}\right) M_{k}\left(\mathbf{n}+\mathbf{e}_{k}\right) \mathbf{1}_{\left\{n_{k}<C_{k}\right\}}+\sum_{k=1}^{K} \boldsymbol{\pi}\left(\mathbf{n}-\mathbf{e}_{k}\right) \lambda_{k} \mathbf{1}_{\left\{n_{k}>0\right\}},
\end{aligned}
$$


with $M_{k}(\mathbf{n})$ the $M \times M$ diagonal matrix with diagonal elements $\mu_{k}(\mathbf{n}, j)$, with $I_{M}$ the $M \times M$ identity matrix and with $A$ the generator matrix of $M(t)$.

\subsection{Regular perturbation}

In the following subsections it is shown that a series expansion approach allows for evaluating the performance of the system under either light-traffic or overload conditions. In particular, it is shown that the series expansion of the stationary solution of the Markov process is regular (i.e., we have a regular perturbation) $[32,34,44]$ and that the computational complexity of calculating the consecutive terms in the series expansion is far better than the computational complexity of calculating the stationary distribution directly. Prior to introducing the equations for the system at hand, we outline the main ideas of the methodology.

The system of equations (1) takes the generic form

$$
\pi Q=0,
$$

where $\boldsymbol{\pi}$ is a vector which collects all stationary probabilities $\pi(\mathbf{n}, j)$ and where $Q$ is a known generator matrix whose off-diagonal elements are the transition rates between states. The row sums of the generator matrix are zero, and the matrix has negative diagonal elements and non-negative off-diagonal elements. Assume now that the entries of the generator matrix are affine functions of a system parameter $\epsilon$. In the following sections, this parameter will be the global arrival rate $\lambda$ for the light-traffic approximation and the global service rate $\mu$ for the overload approximation. As the entries of the generator matrix are affine functions of $\epsilon$, the generic equation (3) can be written as

$$
\boldsymbol{\pi}^{(\epsilon)} Q=\boldsymbol{\pi}^{(\epsilon)}\left(Q^{(\mathbf{0})}+\epsilon Q^{(\mathbf{1})}\right)=0 .
$$

Here we have made the dependence of the stationary solution $\boldsymbol{\pi}$ on $\epsilon$ explicit. Moreover, note that $Q^{(\mathbf{0})}$ is a proper generator matrix: this is the generator matrix of the system for $\epsilon=0$. Now, assume that this Markov process is a uni-chain (the Markov process has at most one ergodic class). In this case, $\boldsymbol{\pi}^{(0)} Q^{(\mathbf{0})}=0$ has a unique normalised solution. Moreover, by Cramer's rule, one easily finds that $\boldsymbol{\pi}^{(\epsilon)}$ is an analytic function of $\epsilon$ in an open interval around $\epsilon=0$. Therefore, let $\boldsymbol{\pi}_{i}$ be the $i$ th term in the series expansion of $\boldsymbol{\pi}^{(\epsilon)}$,

$$
\boldsymbol{\pi}^{(\epsilon)}=\sum_{i=0}^{\infty} \boldsymbol{\pi}_{i} \epsilon^{i} .
$$

Plugging the series expansion (5) into (4) and identifying equal powers of $\epsilon$, we get

$$
\boldsymbol{\pi}_{0} Q^{(\mathbf{0})}=0, \quad \boldsymbol{\pi}_{i+1} Q^{(\mathbf{0})}=-\boldsymbol{\pi}_{i} Q^{(\mathbf{1})} .
$$

Complementing the former set of equations with the normalisation condition,

$$
\boldsymbol{\pi}_{0} \mathbf{e}^{\prime}=1, \quad \boldsymbol{\pi}_{i} \mathbf{e}^{\prime}=0,
$$


for $i>0$, allows for recursively calculating the terms of the series expansion.

For a generic matrix $Q^{(0)}$, there is no gain in computational complexity as one still needs to invert this matrix while solving for the next term in the series expansion. However, for the queueing system at hand, $Q^{(0)}$ has additional structure. Indeed, for the light-traffic approximation, non- $\lambda$ transitions are either departures or changes of the channel state. Assuming a proper ordering of the states of the Markov process, the generator matrix $Q^{(\mathbf{0})}$ is block upper-diagonal, the blocks having the size of the state space of the channel. For the overload approximation, non- $\mu$ transitions are either arrivals or changes of the channel state and with a proper ordering of the state space - a similar block upper-diagonal structure is obtained. In either case, recursively solving the systems of equations (7) is considerably less involved. The equation

$$
\pi_{0} Q^{(0)}=0
$$

reduces to a system of $M$ equations of $M$ unknowns, while for each $i$ the unknowns in the system

$$
\boldsymbol{\pi}_{i+1} Q^{(\mathbf{0})}=-\boldsymbol{\pi}_{i} Q^{(\mathbf{1})}
$$

can be solved in blocks of $M$ unknowns at a time due to the block upper-diagonal structure of $Q^{(\mathbf{0})}$.

\subsection{Overload-traffic analysis}

We first consider the balance equation for $\mu \rightarrow 0$. In particular we express the service rates as

$$
\mu_{k}(\mathbf{n}, j)=\mu \tilde{\mu}_{k}(\mathbf{n}, j)
$$

and consider the Maclaurin series expansion in $\mu$ of the steady-state probabilities:

$$
\boldsymbol{\pi}(\mathbf{n})=\sum_{i=0}^{\infty} \boldsymbol{\pi}_{i}(\mathbf{n}) \mu^{i}
$$

For ease of notation, let $\tilde{M}_{k}(\mathbf{n})=\mu^{-1} M_{k}(\mathbf{n})$. Note that $\tilde{M}_{k}(\mathbf{n})$ does not depend on $\mu$. Plugging the former expression into equation (2) and comparing terms in $\mu^{i}$, we can express the $i$ th order terms in terms of $(i-1)$ st and $i$ th order terms as follows,

$$
\begin{aligned}
\boldsymbol{\pi}_{i}(\mathbf{n}) \sum_{k=1}^{K} \lambda_{k} \mathbf{1}_{\left\{n_{k}<C_{k}\right\}}-\boldsymbol{\pi}_{i}(\mathbf{n}) A= & \mathbf{1}_{\{i>0\}} \sum_{k=1}^{K} \boldsymbol{\pi}_{i-1}\left(\mathbf{n}+\mathbf{e}_{k}\right) \tilde{M}_{k}\left(\mathbf{n}+\mathbf{e}_{k}\right) \mathbf{1}_{\left\{n_{k}<C_{k}\right\}} \\
& -\mathbf{1}_{\{i>0\}} \boldsymbol{\pi}_{i-1}(\mathbf{n}) \sum_{k=1}^{K} \tilde{M}_{k}(\mathbf{n}) \mathbf{1}_{\left\{n_{k}>0\right\}}+\sum_{k=1}^{K} \boldsymbol{\pi}_{i}\left(\mathbf{n}-\mathbf{e}_{k}\right) \lambda_{k} \mathbf{1}_{\left\{n_{k}>0\right\}} .
\end{aligned}
$$

Plugging $\mathbf{n}=\mathbf{0}=[0,0, \ldots, 0]$ and $i=0$ into the former equation and post-multiplying with $\mathbf{e}^{\prime}$ leads to

$$
\boldsymbol{\pi}_{0}(\mathbf{0}) \mathbf{e}^{\prime}=0
$$

which implies $\boldsymbol{\pi}_{0}(\mathbf{0})=\mathbf{0}$ as the elements of $\boldsymbol{\pi}_{0}(\mathbf{0})$ are non-negative. Using the same arguments, one then shows by iteration that for all $\mathbf{n} \in \mathcal{C} \backslash\{\mathbf{c}\}$, we have $\boldsymbol{\pi}_{0}(\mathbf{n})=\mathbf{0}$ and $\boldsymbol{\pi}_{0}(\mathbf{c}) A=0$. Together with the 
normalisation condition $\sum_{\mathbf{n} \in \mathcal{C}} \boldsymbol{\pi}_{0}(\mathbf{n}) \mathbf{e}^{\prime}=1$, this shows that $\boldsymbol{\pi}_{0}(\mathbf{c})=\mathbf{a}$, the steady-state solution of the Markov process $M(t)$ (i.e., the normalised solution of $\mathbf{a} A=\mathbf{0}$ ).

For the higher-order terms $(i>0)$, we have

$$
\begin{aligned}
\boldsymbol{\pi}_{i}(\mathbf{n})\left(\sum_{k=1}^{K} \lambda_{k} \mathbf{1}_{\left\{n_{k}<C_{k}\right\}} I_{M}-A\right)=\sum_{k=1}^{K} \boldsymbol{\pi}_{i-1}\left(\mathbf{n}+\mathbf{e}_{k}\right) \tilde{M}_{k}\left(\mathbf{n}+\mathbf{e}_{k}\right) \mathbf{1}_{\left\{n_{k}<C_{k}\right\}} \\
\\
+\sum_{k=1}^{K}\left(\boldsymbol{\pi}_{i}\left(\mathbf{n}-\mathbf{e}_{k}\right) \lambda_{k}-\boldsymbol{\pi}_{i-1}(\mathbf{n}) \tilde{M}_{k}(\mathbf{n})\right) \mathbf{1}_{\left\{n_{k}>0\right\}} .
\end{aligned}
$$

For $\mathbf{n} \neq \mathbf{c}$, the matrix on the left-hand side is invertible. Hence, we can calculate the probabilities $\boldsymbol{\pi}_{i}(\mathbf{n})$ in lexicographical order. For $\mathbf{n}=\mathbf{c}$, we get

$$
\boldsymbol{\pi}_{i}(\mathbf{c}) A=\sum_{k=1}^{K}\left(-\boldsymbol{\pi}_{i}\left(\mathbf{c}-\mathbf{e}_{k}\right) \lambda_{k}+\boldsymbol{\pi}_{i-1}(\mathbf{c}) \tilde{M}_{k}(\mathbf{c})\right),
$$

and the matrix on the left-hand side is not invertible. A solution of this equation takes the form

$$
\boldsymbol{\pi}_{i}(\mathbf{c})=\sum_{k=1}^{K}\left(-\boldsymbol{\pi}_{i}\left(\mathbf{c}-\mathbf{e}_{k}\right) \lambda_{k}+\boldsymbol{\pi}_{i-1}(\mathbf{c}) \tilde{M}_{k}(\mathbf{c})\right) A^{\#}+\kappa_{i} \mathbf{a},
$$

for any $\kappa_{i}$. Here, $A^{\#}=\left(A+\mathbf{e}^{\prime} \mathbf{a}\right)^{-1}-\mathbf{e}^{\prime} \mathbf{a}$ is the group inverse of $A$. Finally, the remaining unknown $\kappa_{i}$ follows from the normalisation condition

$$
\sum_{\mathbf{n} \in \mathcal{C}} \boldsymbol{\pi}_{i}(\mathbf{n}) \mathbf{e}^{\prime}=0
$$

In view of the calculations above, one easily verifies that the numerical complexity of the algorithm for the $L$ th order expansion is $O\left(L M^{2} S\right)$ as there are $S / M$ blocks, $L$ terms in the recursion and the operations with blocks have complexity $O\left(M^{3}\right)$.

\subsection{Light-traffic analysis}

Similar arguments can be developed for the case of light-traffic conditions, that is, we set $\lambda_{k}=\lambda \tilde{\lambda}_{k}$ and consider an expansion of the form

$$
\boldsymbol{\pi}(\mathbf{n})=\sum_{i=0}^{\infty} \boldsymbol{\pi}_{i}(\mathbf{n}) \lambda^{i}
$$

In view of the balance equations, the terms of this series expansion adhere

$$
\begin{aligned}
\boldsymbol{\pi}_{i}(\mathbf{n})\left(\sum_{k=1}^{K} M_{k}(\mathbf{n}) \mathbf{1}_{\left\{n_{k}>0\right\}}-A\right) & =\sum_{k=1}^{K} \boldsymbol{\pi}_{i}\left(\mathbf{n}+\mathbf{e}_{k}\right) M_{k}\left(\mathbf{n}+\mathbf{e}_{k}\right) \mathbf{1}_{\left\{n_{k}<C_{k}\right\}} \\
& -\mathbf{1}_{\{i>0\}} \boldsymbol{\pi}_{i-1}(\mathbf{n}) \sum_{k=1}^{K} \tilde{\lambda}_{k} \mathbf{1}_{\left\{n_{k}<C_{k}\right\}}+\mathbf{1}_{\{i>0\}} \sum_{k=1}^{K} \boldsymbol{\pi}_{i-1}\left(\mathbf{n}-\mathbf{e}_{k}\right) \tilde{\lambda}_{k} \mathbf{1}_{\left\{n_{k}>0\right\}} .
\end{aligned}
$$

For $i=0$, we can show that $\boldsymbol{\pi}_{0}(\mathbf{n})=\mathbf{0}$ for $\mathbf{n} \neq \mathbf{0}$ and $\boldsymbol{\pi}_{0}(\mathbf{0})=\mathbf{a}$. For $i>0$ and $\mathbf{n} \neq \mathbf{0}$, we can recursively calculate all $\boldsymbol{\pi}_{i}(\mathbf{n})$ in reverse lexicographical order as the matrix on the left-hand side is invertible. For 
$\mathbf{n}=\mathbf{0}$, we get

$$
\left.\boldsymbol{\pi}_{i}(\mathbf{0})=-\sum_{k=1}^{K} \boldsymbol{\pi}_{i}\left(\mathbf{e}_{k}\right) M_{k}\left(\mathbf{e}_{k}\right)+\boldsymbol{\pi}_{i-1}(\mathbf{0}) \sum_{k=1}^{K} \tilde{\lambda}_{k}\right) A^{\#}+\tilde{\kappa}_{i} \mathbf{a},
$$

where $\tilde{\kappa}_{i}$ can be determined from the normalisation condition (14).

\subsection{Performance metrics}

In order to quantify the performance of the system at hand we consider the following metrics for the $k$ th queue: the mean queue content $\mathrm{E}\left[Q_{k}\right]$, the variance of the queue content $\operatorname{var}\left[Q_{k}\right]$ and the blocking probability $b_{k}$. As Poisson arrivals see time averages, the blocking probability for the $k$ th queue is the probability that the number of packets in this queue is equal to the queue capacity, $b_{k}=\mathrm{P}\left[n_{k}=C_{k}\right]$. Additionally, we calculate the mean of the total system content $\mathrm{E}\left[Q^{\text {total }}\right]$. These characteristics can be expressed in terms of the stationary distribution $\boldsymbol{\pi}(\mathbf{n})$. We then find approximations of these metrics by replacing the stationary distribution by its $L$ th order expansion,

$$
\begin{aligned}
\mathrm{E}\left[Q^{\text {total }}\right] & =\sum_{\mathbf{n} \in \mathcal{C}} \boldsymbol{\pi}^{(\epsilon)}(\mathbf{n}) \mathbf{e}^{\prime} \mathbf{n} \mathbf{e}^{\prime} \approx \sum_{i=0}^{L} \sum_{\mathbf{n} \in \mathcal{C}} \boldsymbol{\pi}_{i}^{(\epsilon)}(\mathbf{n}) \mathbf{e}^{\prime} \mathbf{n} \mathbf{e}^{\prime} \epsilon^{i}, \\
\mathrm{E}\left[Q_{k}\right] & =\sum_{\mathbf{n} \in \mathcal{C}} \boldsymbol{\pi}^{(\epsilon)}(\mathbf{n}) \mathbf{e}^{\prime} \mathbf{n} \mathbf{e}_{k}^{\prime} \approx \sum_{i=0}^{L} \sum_{\mathbf{n} \in \mathcal{C}} \boldsymbol{\pi}_{i}^{(\epsilon)}(\mathbf{n}) \mathbf{e}^{\prime} \mathbf{n} \mathbf{e}_{k}^{\prime} \epsilon^{i}, \\
\operatorname{var}\left[Q_{k}\right] & =\sum_{\mathbf{n} \in \mathcal{C}} \boldsymbol{\pi}^{(\epsilon)}(\mathbf{n}) \mathbf{e}^{\prime}\left(\mathbf{n} \mathbf{e}_{k}^{\prime}\right)^{2}-\mathrm{E}\left[Q_{k}\right]^{2} \approx \sum_{i=0}^{L} \sum_{\mathbf{n} \in \mathcal{C}} \boldsymbol{\pi}_{i}^{(\epsilon)}(\mathbf{n}) \mathbf{e}^{\prime}\left(\mathbf{n} \mathbf{e}_{k}^{\prime}\right)^{2} \epsilon^{i}-\mathrm{E}\left[Q_{k}\right]^{2}, \\
b_{k} & =\sum_{\mathbf{n} \in \mathcal{C}} \boldsymbol{\pi}^{(\epsilon)}(\mathbf{n}) \mathbf{e}^{\prime} \mathbf{1}_{\left\{\mathbf{n} \mathbf{e}_{k}^{\prime}=C_{k}\right\}} \approx \sum_{i=0}^{L} \sum_{\mathbf{n} \in \mathcal{C}} \boldsymbol{\pi}_{i}^{(\epsilon)}(\mathbf{n}) \mathbf{e}^{\prime} \epsilon^{i} \mathbf{1}_{\left\{\mathbf{n e}_{k}^{\prime}=C_{k}\right\}},
\end{aligned}
$$

where the dependence on $\epsilon$ has again been made explicit, $\epsilon$ being the service rate $\mu$ in the overload expansion or the arrival rate $\lambda$ for the light-traffic case.

\section{Modelling the wireless environment}

In this section, we introduce a particular channel model for the multi-channel wireless communication scenario at hand. For a wide range of applications, fluctuations in a wireless communication link can be efficiently modelled by means of Markov chains. For a single channel, multiple authors have proposed Markovian models before, see e.g. [29, 45-47]. We here adapt the discrete-time channel model of [29] to the continuous-time Markov chain setting.

We also investigate the influence of cross-channel correlation, and therefore require a Markovian channel model with cross-channel correlation. While many authors have proposed models with cross-channel correlation [58-60], none of these models fit the present Markovian framework. Therefore, we extend the single-channel approach of [29] to multiple correlated channels. The proposed approach for both single- and 
multi-channel models also extends to other channel models including Rician fading channels and Nakagami$m$ fading channels [18].

\subsection{Single-channel model}

To model the behaviour of a single Rayleigh fading channel by a Markov chain with state space $\mathcal{J}=$ $\{1,2, \ldots, J\}$, we construct a Markov chain such that the stationary distribution of the Markov model closely matches the distribution of the Signal-to-Noise ratio (SNR), as well as the rates in which certain levels are crossed.

For a Rayleigh fading channel, the stationary distribution of the SNR is exponentially distributed,

$$
\mathrm{P}[\mathrm{SNR} \leq \eta]=F(\eta)=1-\exp \left(-\frac{\eta}{\nu}\right),
$$

where $\nu$ denotes the mean SNR. To closely match this distribution, we first discretise the distribution. That is, we choose levels $\eta_{0}<\eta_{1}<\eta_{2}<\ldots<\eta_{J}$, and define,

$$
y_{j}=\mathrm{P}\left[\eta_{j-1}<\mathrm{SNR} \leq \eta_{j}\right]=F\left(\eta_{j}\right)-F\left(\eta_{j-1}\right)=\exp \left(-\frac{\eta_{j-1}}{\nu}\right)-\exp \left(-\frac{\eta_{j}}{\nu}\right) .
$$

That is, $y_{j}$ is the probability that the SNR lies in the interval $\left(\eta_{j-1}, \eta_{j}\right]$.

The time variations of the SNR are characterised by the Doppler frequency effect, which is caused by the motion of the mobile nodes. In particular, let $N_{j}$ denote the mean number of times per time unit the SNR crosses the threshold $\eta_{j}$ downward. Obviously, $N_{j}$ is also the mean number of times per time unit the SNR crosses the threshold $\eta_{j}$ upward. $N_{j}$ is given by [48],

$$
N_{j}=\sqrt{\frac{2 \pi \eta_{j}}{\nu}} f \exp \left(-\frac{\eta_{j}}{\nu}\right)
$$

where $f$ denotes the Doppler frequency and where $\nu$ is the mean SNR as before.

We now construct a Markov chain $Y(t)$ with stationary distribution $\left\{y_{j}, j \in \mathcal{J}\right\}$ such that the mean number of transitions per time unit from state $j$ to state $j+1$ equals $N_{j}$. Indeed, a transition from state $j$ to state $j+1$ corresponds to crossing threshold $\eta_{j}$. Downward crossings are defined likewise. This leads to the following transition rates for $j, k \in \mathcal{J}$,

$$
a_{j, k}= \begin{cases}\frac{N_{j}}{y_{j}} & \text { for } k=j+1 ; \\ \frac{N_{j-1}}{y_{j}} & \text { for } k=j-1 ; \\ 0 & \text { otherwise. }\end{cases}
$$

It is now easily verified by checking the local balance equations that $\left\{y_{j}, j \in \mathcal{J}\right\}$ is the stationary distribution of the Markov chain with transition rates $\left\{a_{j, k}, j, k \in \mathcal{J}\right\}$. Hence we have found a Markov process which agrees with the (discretised) stationary distribution, as well as with the level crossings specified by the Doppler effect. 
Once the generator matrix $\tilde{A}$ with transition rates $a_{j, k}$ is obtained for a single channel as above, we can easily expand the model for the case of multiple independent channels. We can do so by merging $K$ single-channel models, the $k$ th model having state space $\mathcal{J}_{k}=\left\{1, \ldots, J_{k}\right\}$ into a multidimensional Markov process. The state space of the joint model is then $\mathcal{M}=\mathcal{J}_{1} \times \ldots \mathcal{J}_{K}$. In other words, the state of the channels is described by a vector, the $k$ th element in this vector denoting the state of the $k$ th channel. The generator matrix of this Markov chain is,

$$
A=\bigoplus_{k=1}^{K} A_{k},
$$

where $A_{k}$ is the $J_{k} \times J_{k}$ generator matrix of the $k$ th channel and where $\oplus$ denotes the Kronecker sum.

\subsection{Multiple channels}

In order to assess the effects of channel correlations, we now construct a channel model where (i) the characteristics of the single-channel models are given and (ii) some form of correlation between the channels is introduced. Moreover, our channel model with cross-correlation will have the same state space as a similar model without correlation.

Copulas. We consider a model with $K$ Rayleigh fading channels. Let $\nu_{k}$ denote the mean SNR of the $k$ th channel and let $f_{k}$ denote the Doppler frequency of the $k$ th channel. Given the distribution $F_{k}$ of the SNR of the $k$ th channel for $k=1, \ldots, K$, we construct a joint distribution of the channel SNRs by means of a copula. A $K$-dimensional copula $C(\mathbf{x}), \mathbf{x} \in[0,1]^{K}$, is a multivariate probability distribution for which the marginal probability distribution of each variable is uniform, see $[49,50]$. Hence, for a copula one has,

$$
C(1, \ldots, 1, u, 1, \ldots, 1)=u
$$

for $0 \leq u \leq 1$. Given a copula $C_{\theta}$ with parameter $\theta$, the $K$-dimensional distribution

$$
F(\mathbf{x}, \theta)=C_{\theta}\left(F_{1}\left(x_{1}\right), \ldots, F_{K}\left(x_{K}\right)\right)
$$

then has the required marginal distributions. The parameter $\theta$ can then be used to introduce the required amount of cross-channel correlation. For the numerical examples, we adopt the Clayton and Vine copulas.

The Clayton copula is an Archimedean copula with generator $\phi(x)=\max \left((1+\theta x)^{-1 / \theta}, 0\right)$, yielding the copula,

$$
C_{\theta}(\mathbf{x})=\max \left(\sum_{k=1}^{K} x_{k}^{-\theta}-(K-1), 0\right)^{-1 / \theta}
$$

for $\mathbf{x}=\left[x_{1}, \ldots, x_{K}\right] \in\left(\mathbb{R}^{+}\right)^{K}$ and $\theta \in[-1 /(d-1), \infty) \backslash\{0\}$. For the Rayleigh fading channels we therefore get,

$$
F(\mathbf{x}, \theta)=\max \left(\sum_{k=1}^{K}\left(1-\exp \left(-x_{k} / \nu_{k}\right)\right)^{-\theta}-(K-1), 0\right)^{-1 / \theta}
$$


By construction, the Clayton copula is symmetric. To allow for a richer correlation structure, one can construct a Vine copula. Vine copulas combine multiple bivariate copulas into a single multivariate copula. Such a pair-copula decomposition allows for a flexible and intuitive way of extending bivariate copulas to higher dimensions [49-52]. For the general theory on Vine copulas, we refer to [52]. We will apply a 3dimensional regular D-Vine in the remainder. The density of the multivariate distribution is then given by,

$$
f\left(x_{1}, x_{2}, x_{3}\right)=f_{1}\left(x_{1}\right) f_{2}\left(x_{2}\right) f_{3}\left(x_{3}\right) c_{12}\left(F_{1}\left(x_{1}\right), F_{2}\left(x_{2}\right)\right) c_{23}\left(F_{2}\left(x_{2}\right), F_{3}\left(x_{3}\right)\right) c_{13 \mid 2}\left(F_{1 \mid 3}\left(x_{1} \mid x_{2}\right), F_{3 \mid 2}\left(x_{3} \mid x_{2}\right)\right)
$$

or, additionally assuming conditional independence, by,

$$
f\left(x_{1}, x_{2}, x_{3}\right)=f_{1}\left(x_{1}\right) f_{2}\left(x_{2}\right) f_{3}\left(x_{3}\right) c_{12}\left(F_{1}\left(x_{1}\right), F_{2}\left(x_{2}\right)\right) c_{23}\left(F_{2}\left(x_{2}\right), F_{3}\left(x_{3}\right)\right) c_{13 \mid 2}\left(F_{1}\left(x_{1}\right), F_{3}\left(x_{3}\right)\right) .
$$

Here each $c_{*}(\cdot, \cdot)$ is a bivariate copula, $F_{k}(\cdot)$ is the univariate distribution of the SNR of the $k$ th channel as above, and $f_{k}(\cdot)$ is the corresponding density function.

Discretisation. We now again discretise the SNRs. Assuming we discretise the SNR of the $k$ th channel in $J_{k}$ levels, let $\eta_{k, 0}<\eta_{k, 1}<\eta_{k, 2}<\ldots<\eta_{k, J_{k}}$ denote the thresholds of these SNR levels. We then introduce the multivariate discrete distribution,

$$
y_{\mathbf{j}}=\mathrm{P}\left[\eta_{1, j_{1}-1}<\mathrm{SNR}_{1} \leq \eta_{1, j_{1}}, \ldots, \eta_{K, j_{K}-1}<\mathrm{SNR}_{K} \leq \eta_{K, j_{K}}\right]
$$

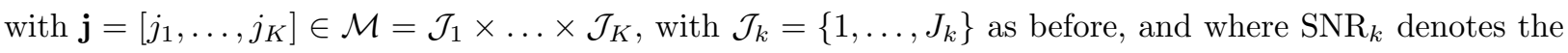
SNR of the $k$ th channel. We can of course express $y_{\mathbf{j}}$ in terms of $F(\mathbf{x})$ as follows,

$$
y_{\mathbf{j}}=\sum_{\mathbf{i} \in\{0,1\}^{K}}(-1)^{\|\mathbf{i}\|} F\left(\eta_{1, j_{1}-i_{1}}, \ldots, \eta_{K, j_{K}-i_{K}}\right),
$$

with $\mathbf{i}=\left[i_{1}, \ldots, i_{K}\right]$ and where $\|\mathbf{i}\|=\sum_{k=1}^{K} i_{k}$ is the $\ell_{1}$ norm. For example, for $K=3$ we have,

$$
\begin{aligned}
y_{j_{1}, j_{2}, j_{3}}=F( & \left.\eta_{1, j_{1}}, \eta_{2, j_{2}}, \eta_{3, j_{3}}\right)-F\left(\eta_{1, j_{1}-1}, \eta_{2, j_{2}}, \eta_{3, j_{3}}\right)-F\left(\eta_{1, j_{1}}, \eta_{2, j_{2}-1}, \eta_{3, j_{3}}\right) \\
& +F\left(\eta_{1, j_{1}-1}, \eta_{2, j_{2}-1}, \eta_{3, j_{3}}\right)-F\left(\eta_{1, j_{1}}, \eta_{2, j_{2}}, \eta_{3, j_{3}-1}\right)+F\left(\eta_{1, j_{1}-1}, \eta_{2, j_{2}}, \eta_{3, j_{3}-1}\right) \\
& +F\left(\eta_{1, j_{1}}, \eta_{2, j_{2}-1}, \eta_{3, j_{3}-2}\right)-F\left(\eta_{1, j_{1}-1}, \eta_{2, j_{2}-1}, \eta_{3, j_{3}-1}\right) .
\end{aligned}
$$

Construction of the Markov process. As for the single-channel case, the time variations of the SNR are characterised by the Doppler frequency effect, which is caused by the motion of the mobile nodes. We now construct a Markov chain such that the mean number of upcrossings of the thresholds for the $k$ th channel is in accordance with the Doppler effect. That is, the mean number of upcrossings of level $\eta_{k, j}$ by the $k$ th channel equals,

$$
N_{k, j}=\sqrt{\frac{2 \pi \eta_{k, j}}{\nu}} f_{k} \exp \left(-\frac{\eta_{k, j}}{\nu}\right) .
$$


Additionally assuming that channels do not cross at the same time, we find for $\mathbf{i}, \mathbf{j} \in \mathcal{M}$,

$$
\alpha_{\mathbf{j}, \mathbf{i}}= \begin{cases}\frac{N_{k, j_{k}}}{y_{\mathbf{j}}} & \text { for } \mathbf{i}=\mathbf{j}+\mathbf{e}_{k} ; \\ \frac{N_{k, j_{k}-1}}{y_{\mathbf{j}}} & \text { for } \mathbf{i}=\mathbf{j}-\mathbf{e}_{k} ; \\ 0 & \text { otherwise }\end{cases}
$$

As for the single-channel case, it is now easily verified by checking the local balance equations that $\left\{y_{\mathbf{j}}, \mathbf{j} \in\right.$ $\mathcal{M}\}$ is the stationary distribution of the Markov chain with transition rates $\left\{\alpha_{\mathbf{j}, \mathbf{i}}, \mathbf{j}, \mathbf{i} \in \mathcal{M}\right\}$. Hence we have found a Markov process which agrees with the (discretised) stationary (multivariate) distribution, as well as with the level crossings specified by the Doppler effect.

\subsection{Channel State Information}

The Markov chains for the single and multiple channels above describe the evolution of the SNR of the channels. It now remains to map the states of these Markov chains on the transmission rates that can be achieved. We describe the mapping for the multivariate case, the single-channel model being a particular example.

The maximal achievable channel capacity $\gamma$ for a given SNR $\eta$ and bandwidth $B$, is given by the ShannonHartley theorem [48],

$$
\gamma=B \log _{2}(1+\eta) .
$$

As the transmission rate is proportional to the channel capacity, we introduce the following mapping $\mathbf{j}=$ $\left[j_{1}, \ldots, j_{K}\right] \rightarrow \mathbf{g}_{\mathbf{j}}$ from $\mathcal{M}$ to $[0,1]^{K}$,

$$
\mathbf{g}_{\mathbf{j}}=\left[\frac{\log _{2}\left(1+\xi_{1, j_{1}}\right)}{\log _{2}\left(1+\eta_{\text {best }}\right)}, \ldots, \frac{\log _{2}\left(1+\xi_{K, j_{K}}\right)}{\log _{2}\left(1+\eta_{\text {best }}\right)}\right],
$$

where $\eta_{\text {best }}$ is the best SNR that is achievable (for all channels) and where $\xi_{k, j}$ is the average SNR of the $k$ th channel in state $j$,

$$
\xi_{k, j}=\frac{\int_{\eta_{k, j-1}}^{\eta_{k, j}} \eta d F_{k}(\eta)}{\eta_{k, j}-\eta_{k, j-1}} .
$$

The $k$ th element $g_{\mathbf{j} k}$ of the vector $\mathbf{g}_{\mathbf{j}}$ denotes the fraction of the maximal transmission rate that is available for the $k$ th channel when the channel state is $\mathbf{j}$. The mapping from SNR to rate depends on the system specifications and can vary from one transmitter to another. The value $g_{\mathbf{j} k}$ is referred to as the channel state information and is made available to the opportunistic scheduler.

\subsection{Examples of schedulers}

To illustrate our approach, we consider several schedulers including purely opportunistic, purely nonopportunistic and weighted schedulers. We describe the different schedulers in detail below. For $j \in \mathcal{M}$, $g_{j k}$ denotes the channel state information of the channel of the $k$ th $\mathrm{MU}$ when the background process is in state $j$. 
MaxRate. A first example of a greedy opportunistic scheduler is the MaxRate scheduler. This scheduler serves the MUs with the best channel conditions. Let $\kappa_{M R}(j)$ be the set

$$
\kappa_{\mathrm{MR}}(j)=\underset{k \in\{1, \ldots, K\}}{\arg \max } g_{j k} .
$$

For MaxRate, we have the following service rate,

$$
\mu_{k}(\mathbf{n}, j)= \begin{cases}\mu g_{j k} \frac{1}{\left|\kappa_{\mathrm{MR}}(j)\right|} & \text { for } k \in \kappa_{\mathrm{MR}}(j) \\ 0 & \text { otherwise. }\end{cases}
$$

Here $\left|\kappa_{M R}(j)\right|$ is the cardinality of $\kappa_{M R}(j)$. This scheduler was first considered in [4] for single-cell MU communication.

MaxWeight. In contrast to MaxRate, the MaxWeight scheduler selects the user with the maximum weight, which is calculated as the product of queue length and channel quality, see [43]. For $\mathbf{N}(t)=\mathbf{n}$ and $M(t)=j$, MaxWeight selects users at time $t$ from the set,

$$
\kappa_{\mathrm{MW}}(\mathbf{n}, j)=\underset{k \in\{1, \ldots, K\}}{\arg \max } g_{j k} n_{k} .
$$

Hence this results in the following service rate for MaxWeight,

$$
\mu_{k}(\mathbf{n}, j)= \begin{cases}\mu g_{j k} \frac{1}{\left|\kappa_{\mathrm{MW}}(j)\right|} & \text { for } k \in \kappa_{\mathrm{MW}}(j) \\ 0 & \text { otherwise. }\end{cases}
$$

Longest connected queue. Schedulers may also not account for the channel state at all. An example of a non-opportunistic scheduler is one that chooses the longest queue. This scheduler is referred to as the Longest Connected Queue (LCQ). It is shown to be stable for dynamic server allocation to parallel queues with randomly varying connectivity in [42]. For $\mathbf{N}(t)=\mathbf{n}$ and $M(t)=\mathbf{j}$, the scheduler serves mobile nodes from the set

$$
\kappa_{\mathrm{LCQ}}=\underset{k \in\{1, \ldots, K\}}{\arg \max } n_{k} .
$$

Notice that also in this case the actual service rate $\mu_{k}(\mathbf{n}, j)$ for node $k$ does depend on the channel condition. Indeed, we have,

$$
\mu_{k}(\mathbf{n}, j)= \begin{cases}\mu g_{j k} \frac{1}{\left|\kappa_{\mathrm{LCQ}}\right|} & \text { for } k \in \kappa_{\mathrm{LCQ}} \\ 0 & \text { otherwise. }\end{cases}
$$

Processor sharing. Finally, we mention two schedulers which are inspired by discriminatory (DPS) and generalised processor sharing (GPS) [34], but with weights set to reflect the channel conditions. The share 
of transmission resources assigned for each user takes one of the following forms:

$$
\begin{aligned}
& \text { DPS: } \mu_{k}(\mathbf{n}, j)=\mu \frac{g_{j k} n_{k}}{\sum_{\ell=1}^{K} g_{j \ell} n_{\ell}}, \\
& \text { GPS: } \mu_{k}(\mathbf{n}, j)=\mu \frac{g_{j k} \mathbf{1}_{\left\{n_{k}>0\right\}}}{\sum_{\ell=1}^{K} g_{j \ell} \mathbf{1}_{\left\{n_{\ell}>0\right\}}} .
\end{aligned}
$$

The formulas for the DPS and GPS schedulers defined above assume that the overall transmission rate is constant over time. This is the case in a scenario where there are no simultaneous transmissions, and the transmission power is adapted to the SNR. Note however that even if there is but one transmission at any time, one does not have non-zero departure rates from the different queues. The departure rate is the product of the departure rate from the queue if it selected and the probability that this queue is selected, and therefore non-zero for every queue which can be selected.

In general, if there are multiple simultaneous transmissions, the departure rates from the different queues will depend both on the queue sizes and on the channel states. The combined transmission rate will also be a function of the queue sizes and the channel states, as transmitting over one channel can influence other channels.

\section{Results}

In this section we compare various schedulers by some numerical examples. We consecutively consider systems with independent channels and systems with cross-channel correlation. The following performance metrics are calculated by means of (17): the mean queue content $\mathrm{E}\left[Q_{k}\right]$, the variance of the queue content $\operatorname{var}\left[Q_{k}\right]$ and the blocking probability $b_{k}$ of the $k$ th queue. Assuming identical queues and channels, we provide results for a single queue, and therefore drop the index $k$ in the figures. As our analysis is an approximation, we validate our results by simulations. The simulation results are obtained by means of the standard Gillespie algorithm, see for example [53].

\subsection{Independent channels}

We first assume that all channels are independent and identical with Doppler frequency $f_{k}=100 \mathrm{~Hz}$ and mean SNR level $\nu_{k}=30 \mathrm{~dB}$ for $k=1, \ldots, K$. To construct the channel model, we partition the SNR into 3 ranges, the thresholds being chosen as follows: $\eta_{0}=0 \mathrm{~dB}, \eta_{1}=23 \mathrm{~dB}, \eta_{2}=27 \mathrm{~dB}, \eta_{3}=40 \mathrm{~dB}$. Note that various strategies of SNR partitioning can be applied in order to establish SNR thresholds, see [54]. With these parameters, following the approach described in subsection 4.1, we find the following generator matrix:

$$
A_{k}=\left(\begin{array}{ccc}
-50.98 & 50.98 & 0 \\
42.99 & -93.39 & 50.39 \\
0 & 17.74 & -17.74
\end{array}\right)
$$


In the figures below, we consider systems with $K=4$ mobile users experiencing independent and stochastically identical channel conditions. Up to $C=10$ packets can be stored at the buffer of the AP for each mobile user. Packets that arrive at a full buffer are rejected. We assume a maximum achievable downlink transmission rate of $10 \mathrm{Mbit} / \mathrm{s}$ and an average packet size of 1.125 MB. Note that with the above assumptions, the channel process with transition rate matrix $A=\oplus_{k=1}^{4} A_{k}$ has $3^{4}$ states, whereas the number of queueing states amounts to $11^{4}$ states. Hence, the Markov process at hand has 1,185,921 states.

Remark 2. We choose a fairly large packet size in the numerical examples. As the size of the state space of the Markov process (and the numerical complexity for solving it) grows quickly with the queue capacity, this capacity cannot be chosen arbitrarily large. Opting for a larger packet size implies that the buffer capacity expressed in bytes is larger as well, without any additional numerical complexity. If the packets are smaller in reality, one can approximate the system with small packets by a system where packets are aggregated into larger. The queue content is then expressed in terms of aggregated packets, and only drops or increases if all packets of the aggregated packet have departed or arrived.

In a first set of figures, we compare the MaxRate, MaxWeight and LCQ schedulers. Figures 2 and 3 show the mean and variance of the queue content as well as the blocking probabilities for these schedulers, in the light-traffic and in the overloaded regime, respectively. For each of these performance measures, we plot the 5th, 15th and 100th order expansions, as well as simulation results for comparison. Both simulation and numerical results indicate that the purely opportunistic MaxRate scheduler performs better than MaxWeight and LCQ in both the light-traffic regime and the overloaded regime. The reason for this advantage can be explained as follows: MaxRate always serves a customer with the maximum available transmission rate, and therefore, maximises the throughput of the system. In contrast, the MaxWeight policy selects the user with the highest product of queue length and transmission rate. Under this strategy the system benefits from the multi-channel diversity while preventing service starvation in the queues with constantly poor channel quality. Fairness of the MaxWeight scheduler, however, comes at the cost of a decrease of the system throughput as compared to the purely opportunistic MaxRate. In comparison with LCQ, MaxWeight performs slightly better than the LCQ scheduler, due to the utilisation of the channel information. Recall that LCQ does not benefit from the channel information and always serves the node with the longest queue, even if the channel conditions are unfavourable for transmission. This approach guarantees fairness, however, impairs the system performance in a transmission environment with a high MU diversity. Numerical results show that although MaxWeight performs better than LCQ, the performance metrics are rather close to each other.

We now assess the accuracy of the series expansions. Obviously, by increasing the order of the expansion, the approximations improve, as they are known to converge to the correct value within the region of convergence of the expansion. While the 5th order expansion is only accurate in a small region around 


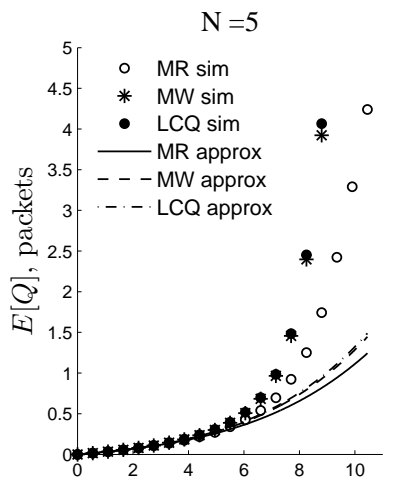

$\lambda, \mathrm{Mbit} / \mathrm{s}$

$\mathrm{N}=5$

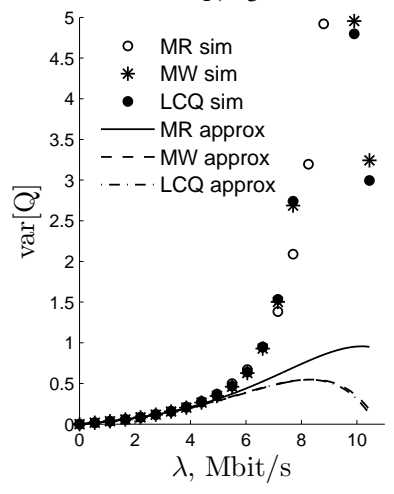

$\mathrm{N}=5$

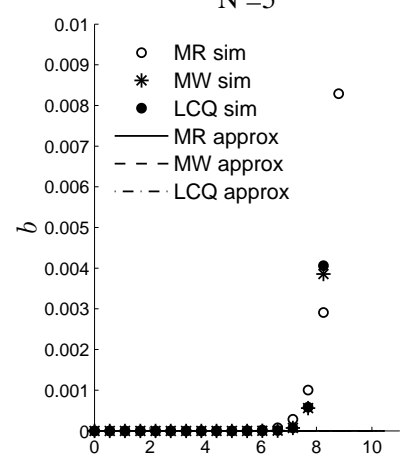

$\lambda, \mathrm{Mbit} / \mathrm{s}$

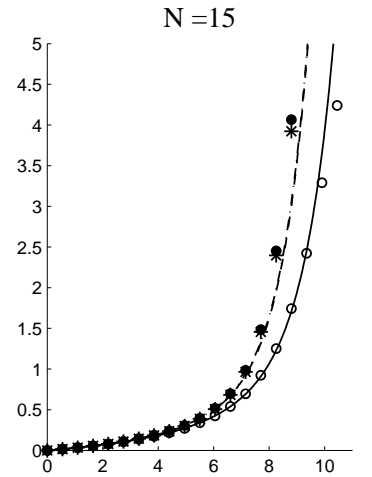

$\lambda, \mathrm{Mbit} / \mathrm{s}$

$\mathrm{N}=15$

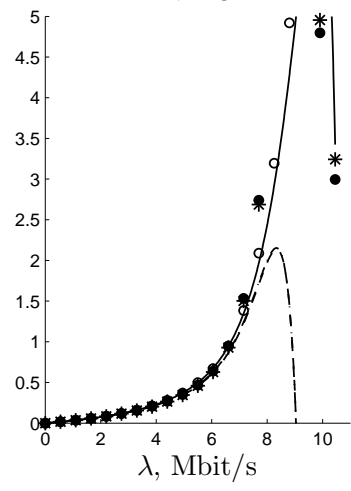

$\mathrm{N}=15$

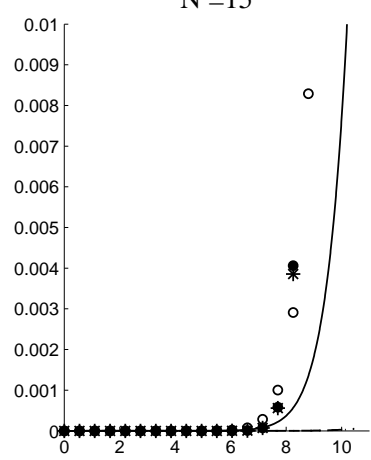

$\lambda, \mathrm{Mbit} / \mathrm{s}$

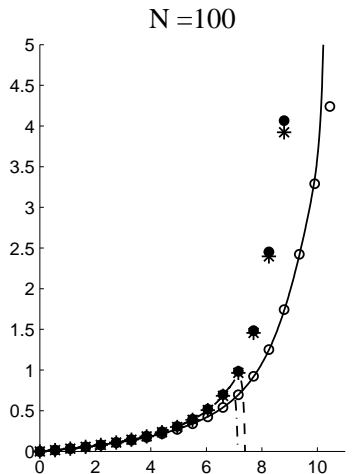

$\lambda, \mathrm{Mbit} / \mathrm{s}$

$\mathrm{N}=100$

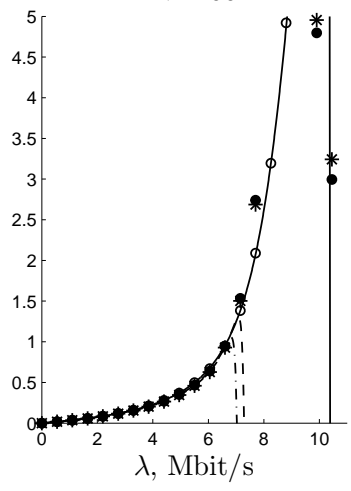

$\mathrm{N}=100$

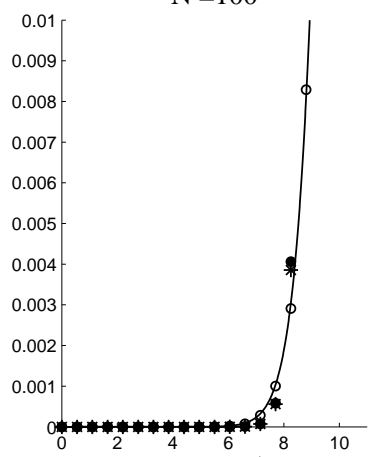

$\lambda, \mathrm{Mbit} / \mathrm{s}$

Figure 2: Mean queue content, variance and blocking probability in the light traffic regime for systems with $K=4$ mobile users, $M=3$ channel states, and for the MaxRate (MR), MaxWeight (MW) and LCQ scheduling disciplines 

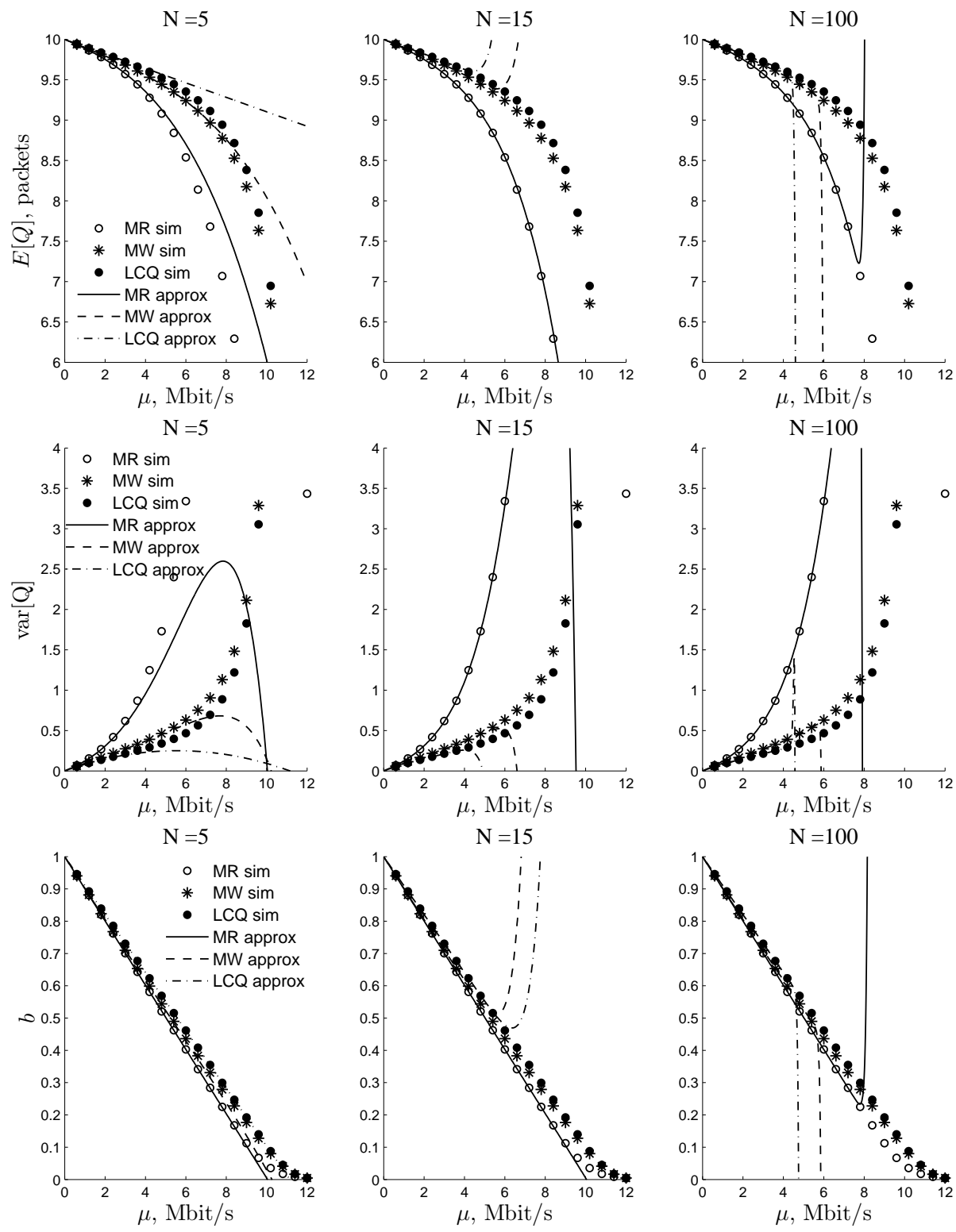

Figure 3: Mean queue content, variance and blocking probability in the overload traffic regime for systems with $K=4$ mobile users, $M=3$ channel states, and for the MaxRate (MR), MaxWeight(MW) and LCQ scheduling disciplines. 
$\lambda=0$ or $\mu=0$, the 15th order approximation is already accurate in a much wider region, while the 100th order expansion is accurate in an even wider region. Note that the series expansions of all performance measures (for a particular scheduler) have the same region of convergence, as they are derived from the same expansion of the steady-state distribution. In fact, the higher order expansions allow for heuristically determining the region of convergence as we get a very accurate match in the region of convergence, followed by an almost immediate and considerable deviation of the true value. This is the case for the 100th order expansion, higher order expansions (not shown here) further confirming that the position of this sudden deviation is fixed. This then indeed strongly suggests that this position corresponds with the boundary of the region of convergence. On the figures, the the regions of convergence for the light traffic approximation are approximately $\lambda \in[0,10] \mathrm{Mbit} / \mathrm{s}$ for MaxRate, $\lambda \in[0,6.3] \mathrm{Mbit} / \mathrm{s}$ for MaxWeight, and $\lambda \in[0,6.8] \mathrm{Mbit} / \mathrm{s}$ for LCQ. For the overload expansions in Figure 3, we observe the convergence regions $\mu \in[0,7.8] \mathrm{Mbit} / \mathrm{s}, \mu \in[0,6] \mathrm{Mbit} / \mathrm{s}$ and $\mu \in[0,4.7] \mathrm{Mbit} / \mathrm{s}$ for the MaxRate, the MaxWeight and the LCQ scheduler, respectively.

As the blocking probability decreases almost linearly for increasing $\mu$, the 5 th order expansion seems rather accurate in a wide region. The match beyond the region of convergence for low order expansions is coincidence and does not further improve by increasing the order of the expansion. Indeed, the higher order expansions reveal the limits of the region of convergence. For the light-traffic approximations, the blocking probability is very small in the region of convergence for the MaxWeight and LCQ schedulers; the values for which the blocking probability starts increasing lie outside the region of convergence. In contrast, for the MaxRate scheduler, we do find a good match in the region where the blocking probabilities start increasing (the interval $\lambda \in[7,9]$ Mbit).

The next set of figures compare the DPS and GPS schedulers. Figures 4 and 5 depict the mean and variance of the queue content and the blocking probability vs. the global arrival rate and vs. the global service rate, respectively. DPS and GPS are opportunistic schedulers that allocate resources among the mobile users for simultaneous transmission. All users with nonempty queues receive a share of the service proportional to their channel qualities. GPS always allocates resources primarily to the users with favourable channel conditions, while DPS also takes into account the queue content of the customers. The comparison of these two schedulers is similar to the MaxRate and MaxWeight comparison. GPS is purely opportunistic and provides better throughput, while DPS guarantees fairness and prevents service starvation for users experiencing poor channel conditions. The numerical results in Figures 4 and 5 indeed confirm these findings, though the difference between GPS and DPS is not as outspoken as the difference between MaxWeight and MaxRate. Moreover, in the light-traffic regime, GPS and DPS hardly differ. This is not unexpected as DPS and GPS only differ once the queue size grows beyond 1, which does not occur frequently in light traffic.

Regarding the accuracy of the approximations, we again observe that increasing the order of the approximations improves the accuracy, in the region of convergence of the series expansions. Compared to the 

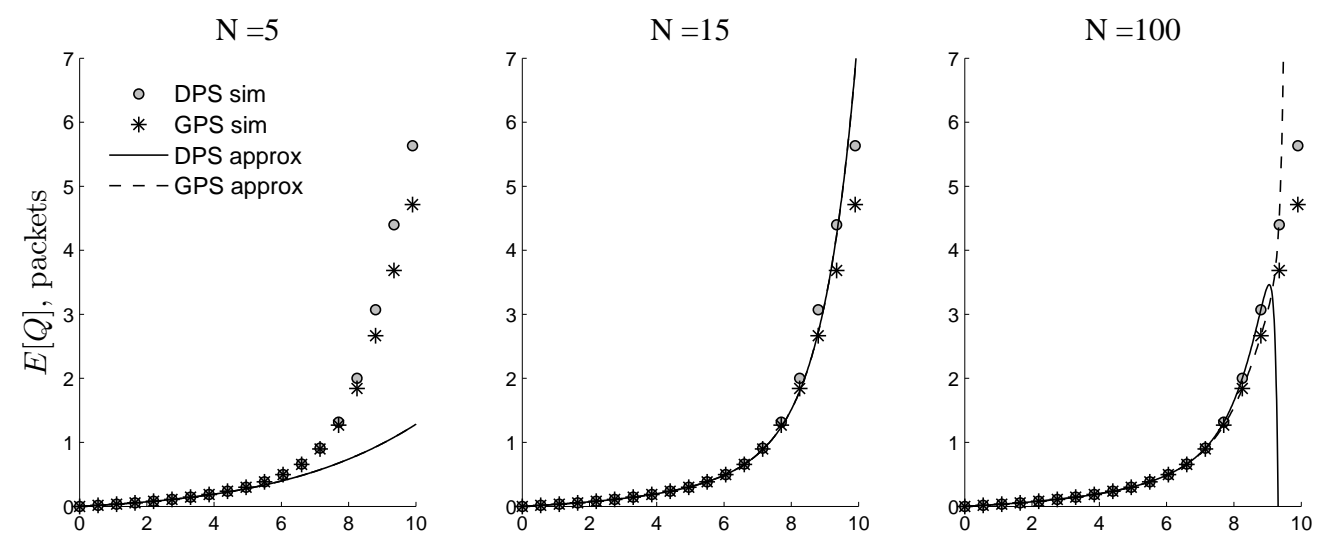

$\lambda, \mathrm{Mbit} / \mathrm{s}$

$\lambda, \mathrm{Mbit} / \mathrm{s}$

$\lambda, \mathrm{Mbit} / \mathrm{s}$

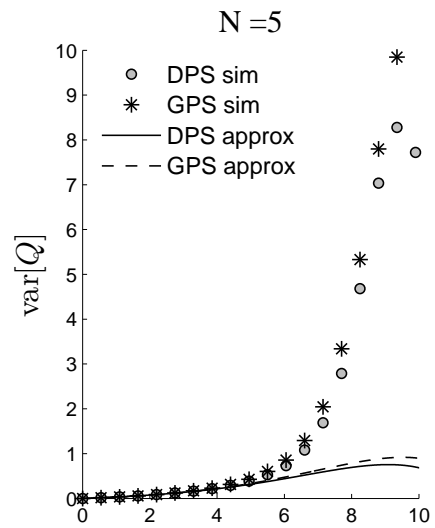

$\mathrm{N}=15$

$$
\mathrm{N}=100
$$
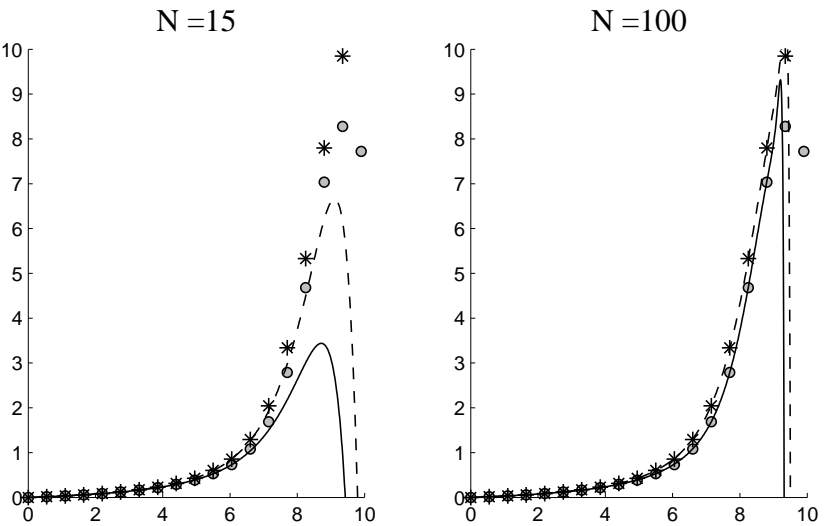

$\lambda, \mathrm{Mbit} / \mathrm{s}$

$\lambda, \mathrm{Mbit} / \mathrm{s}$

$\mathrm{N}=5$

$\mathrm{N}=15$

$\mathrm{N}=100$
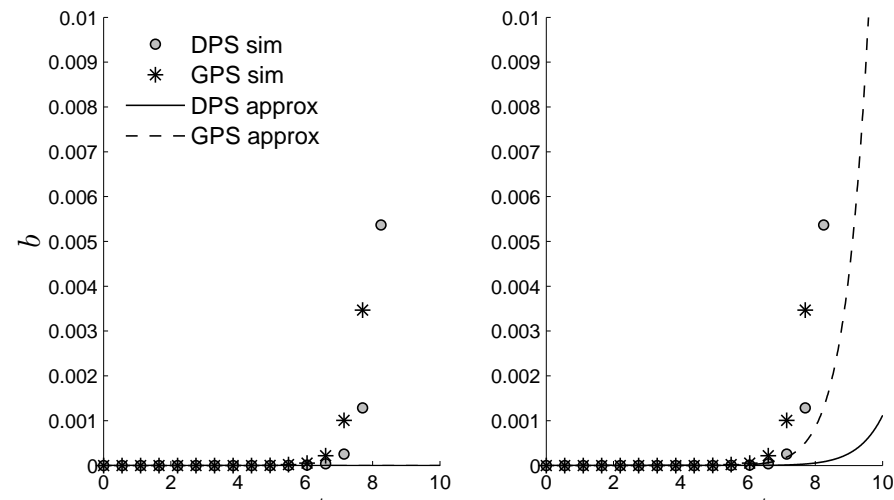

$\lambda, \mathrm{Mbit} / \mathrm{s}$

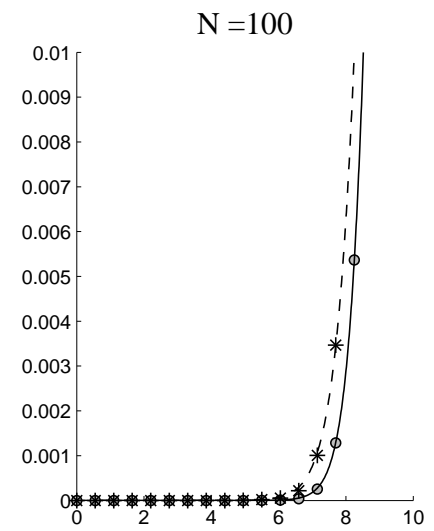

$\lambda, \mathrm{Mbit} / \mathrm{s}$

Figure 4: Mean queue content, variance and blocking probability in the light traffic regime for systems with $K=4$ mobile users, $M=3$ channel states, and for the DPS and GPS scheduling disciplines. 

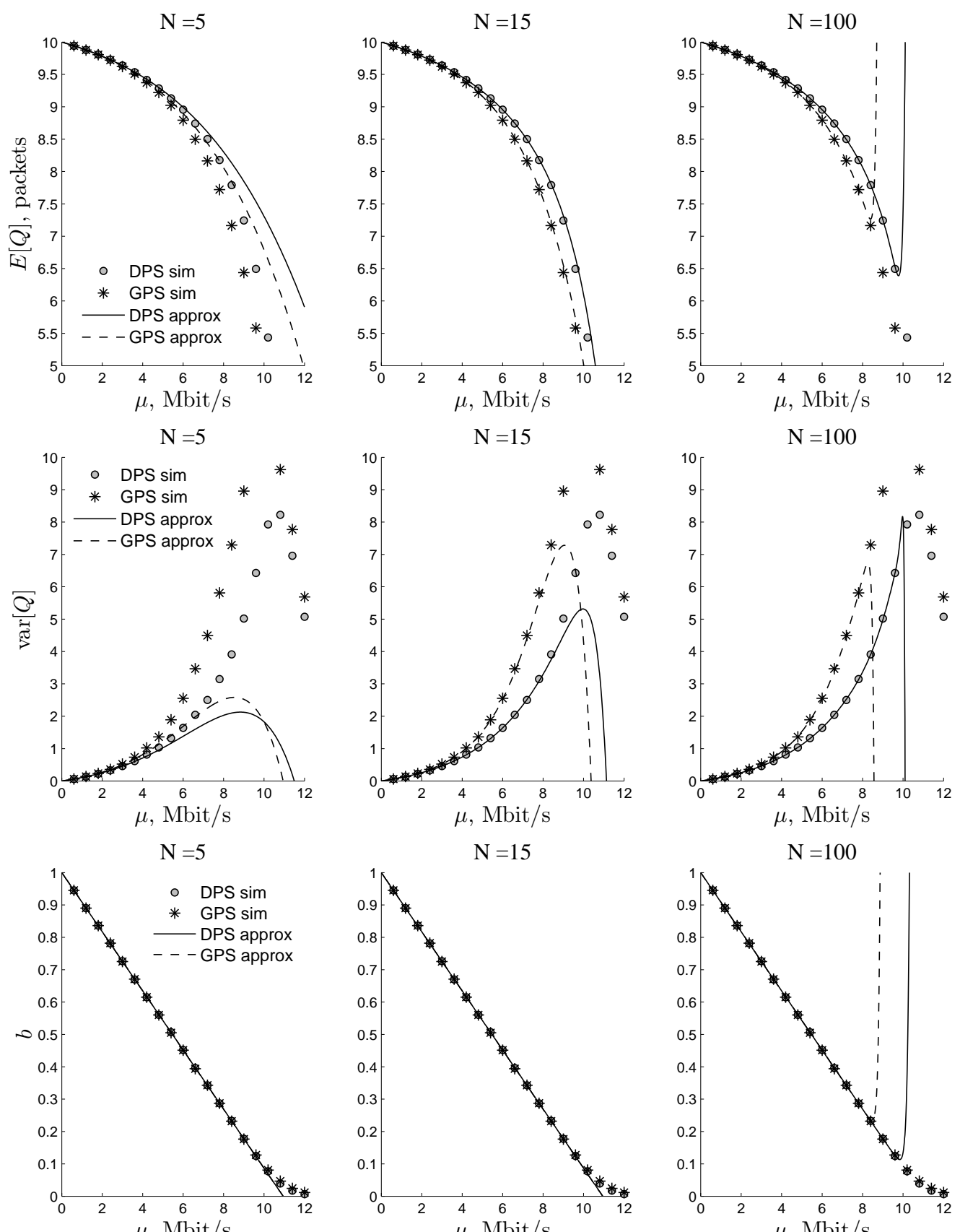

$\mu, \mathrm{Mbit} / \mathrm{s}$

$\mu, \mathrm{Mbit} /$
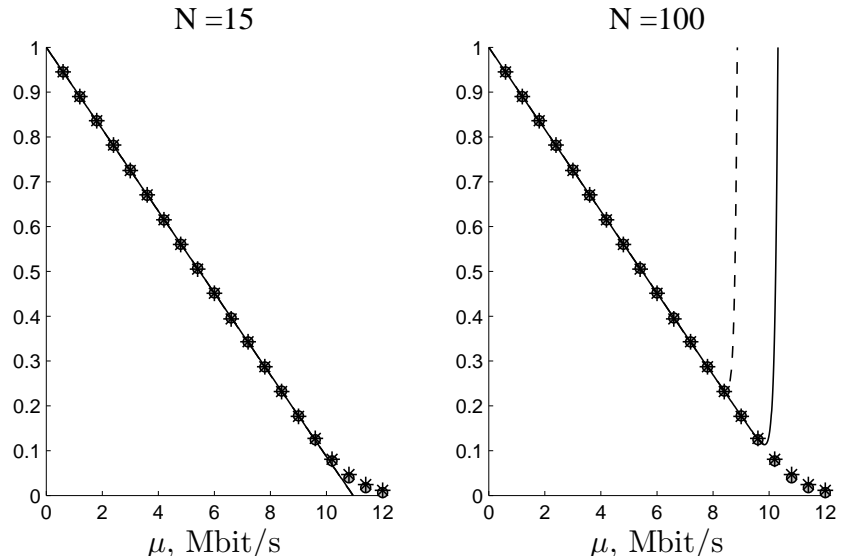

Figure 5: Mean queue content, variance and blocking probability in the overload traffic regime for systems with $K=4$ mobile users and $M=3$ channel states, for the DPS and GPS scheduling disciplines. 
previous plots (figures 2 and 3), the region of convergence is wider. For DPS we have $\lambda \in[0,8.5] \mathrm{Mbit} / \mathrm{s}$ and $\mu \in[0,9.5] \mathrm{Mbit} / \mathrm{s}$, while for GPS, we have $\lambda \in[0,8.5] \mathrm{Mbit} / \mathrm{s}$ and $\mu \in[0,8] \mathrm{Mbit} / \mathrm{s}$.

\subsection{Correlated channels}

We now study opportunistic scheduling with correlated channels. We retain the single channel assumptions of the preceding section: the mean SNR for every channel is $\nu=30 \mathrm{~dB}$, while the Doppler frequency is $f_{k}=100 \mathrm{~Hz}$. We use the threshold values $\eta_{0}=0 \mathrm{~dB}, \eta_{1}=20, \eta_{2}=30 \mathrm{~dB}$, and $\eta_{3}=40 \mathrm{~dB}$. The AP serves 3 mobile users, where each user can store up to $C=30$ packets. Note that we chose a capacity that is considerably larger, compared to the results in section 5.1. Having defined the characteristics of a single channel, we study the following three different correlated channel scenarios.

1. Using a Clayton copula with parameter $\theta=1$, we obtain the following channel correlation matrix,

$$
\mathbf{R}_{1}=\left(\begin{array}{ccc}
1 & 0.369 & 0.369 \\
0.369 & 1 & 0.369 \\
0.369 & 0.369 & 1
\end{array}\right)
$$

where the $k j$ th element of $\mathbf{R}$ denotes Pearson's correlation coefficient of the SNR of the $k$ th and $j$ th channel,

with

$$
r_{k \ell}=\frac{\sigma_{k \ell}^{2}}{\sqrt{\sigma_{k k}^{2} \sigma_{\ell \ell}^{2}}}
$$

$$
\sigma_{k \ell}^{2}=\sum_{\mathbf{j} \in \mathcal{M}} y_{\mathbf{j}} \xi_{k, j_{k}} \xi_{\ell, j_{\ell}}-\left(\sum_{\mathbf{j} \in \mathcal{M}} y_{\mathbf{j}} \xi_{k, j_{k}}\right)\left(\sum_{\mathbf{j} \in \mathcal{M}} y_{\mathbf{j}} \xi_{\ell, j_{\ell}}\right),
$$

where we used the notation of subsection 4.2. For any two channels, we obtain the same correlation. This is not unexpected due to the symmetry of Archimedean copulas.

2. A Vine copula allows for non-symmetric channel correlations. We consider two vine copulas that are build using bivariate Gaussian copulas. We use expression (20), where the copulas $C_{12}, C_{23}$ and $C_{13 \mid 2}$ are Gaussian copulas with correlation coefficients $\rho_{12}=0.2, \rho_{23}=0.1$ and $\rho_{13 \mid 2}=0.3$. This parameter setting results in the following correlation matrix,

$$
\mathbf{R}_{2}=\left(\begin{array}{ccc}
1 & 0.076 & 0.238 \\
0.076 & 1 & 0.152 \\
0.238 & 0.152 & 1
\end{array}\right)
$$

3. The third correlated channel is again based on a Vine copula with Gaussian bivariate copulas, but now with correlation parameters $\rho_{12}=0.8, \rho_{23}=0.7, \rho_{13 \mid 2}=0.6$. For this set of parameters, we get the following correlation matrix,

$$
\mathbf{R}_{3}=\left(\begin{array}{ccc}
1 & 0.565 & 0.650 \\
0.565 & 1 & 0.662 \\
0.650 & 0.662 & 1
\end{array}\right)
$$



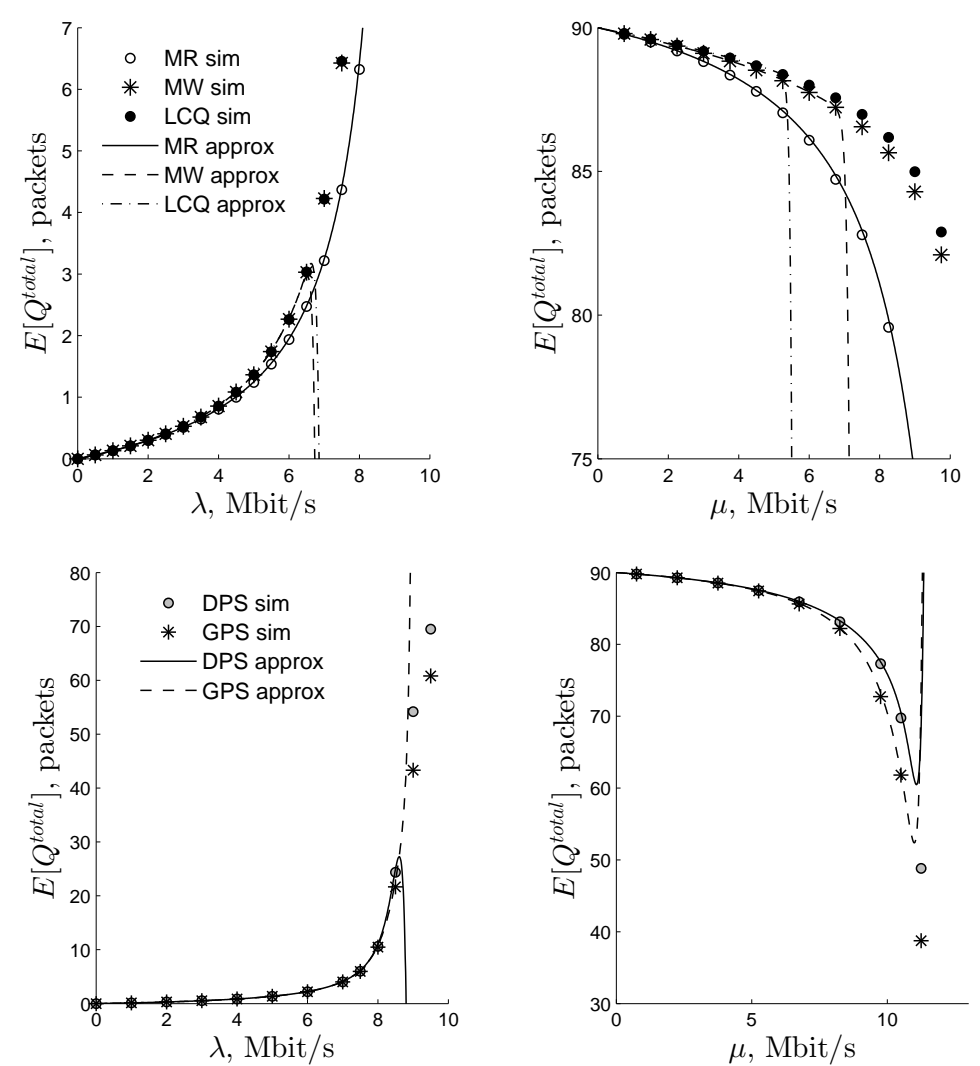

Figure 6: Total buffer content in light and overload traffic for systems with $K=3$ mobile users with correlated channels (Clayton copula) and $M=3$ states per channel, working under MaxRate (MR), MaxWeight (MW), LCQ, DSP and GPS scheduling disciplines. The number of terms in the series expansion is $N=100$.

Figure 6 again compares the MaxRate, MaxWeight and LCQ schedulers as well as the DPS and GPS schedulers. We show the 100th order light-traffic and overload expansions of the total mean queue content, as well as simulation results to verify the accuracy of the approximations. We here use the first correlated channel model with correlation matrix $\mathbf{R}_{1}$. We again obtain a perfect match in a first region, followed by a sharp deviation from the correct value. This again suggests that the region of convergence of the series expansion ends at the position of this fast deviation. Figure 7 depicts the same values for the Vine copula with rate matrix $\mathbf{R}_{2}$.

Positive spatial correlations reduce MU diversity and therefore also reduce the gain of opportunistic scheduling. Nevertheless, if correlations are not strong, the opportunistic approach can still be beneficial. Figure 8 demonstrates the diminished efficiency of opportunistic scheduling by comparing the MaxRate, MaxWeight and LCQ schedulers for independent and correlated channels in the overloaded regime. In particular, Figure 8 compares the differences in total queue content between MaxRate and LCQ, MaxWeight 

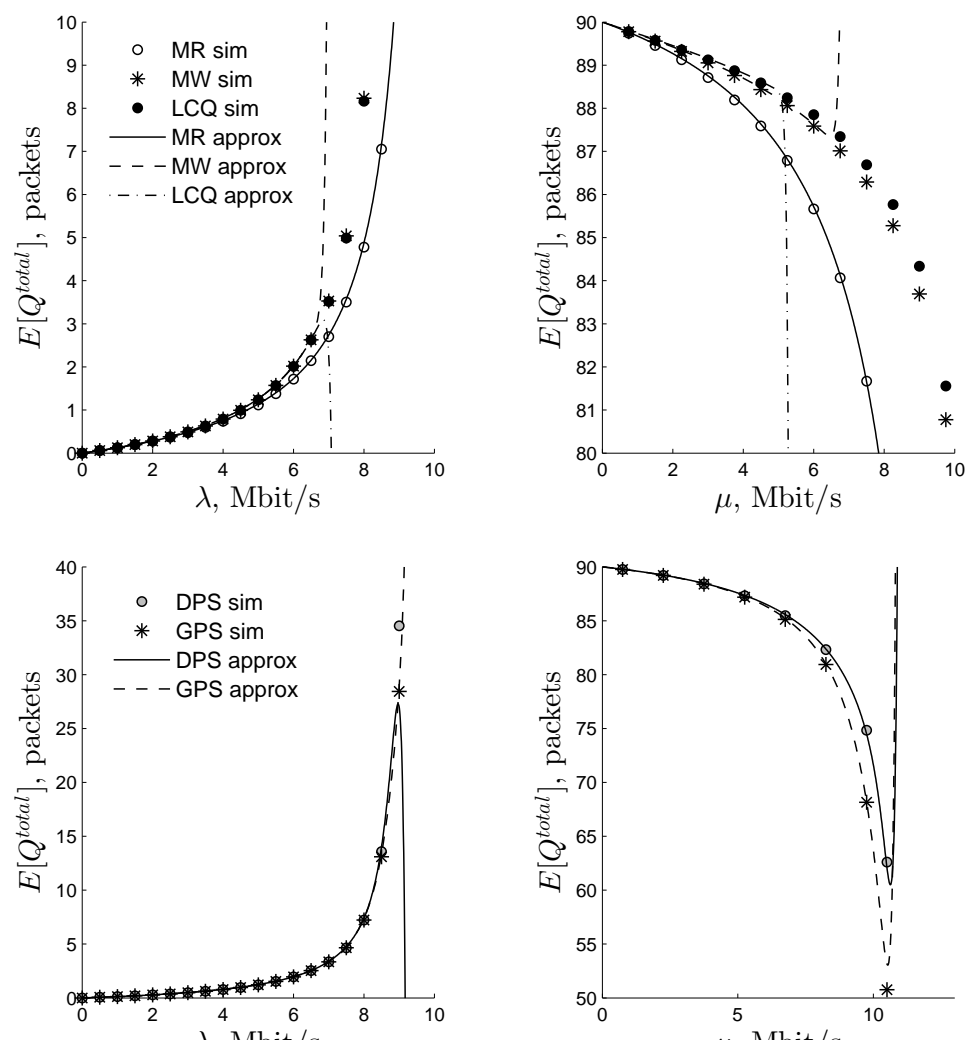

Figure 7: Total buffer content in light and overload traffic for systems with $K=3$ mobile users with correlated channels (Vine copula) and $M=3$ states per channel, working under MaxRate (MR), MaxWeight (MW), LCQ, DSP and GPS scheduling disciplines; the order of the series expansion is $N=100$. 

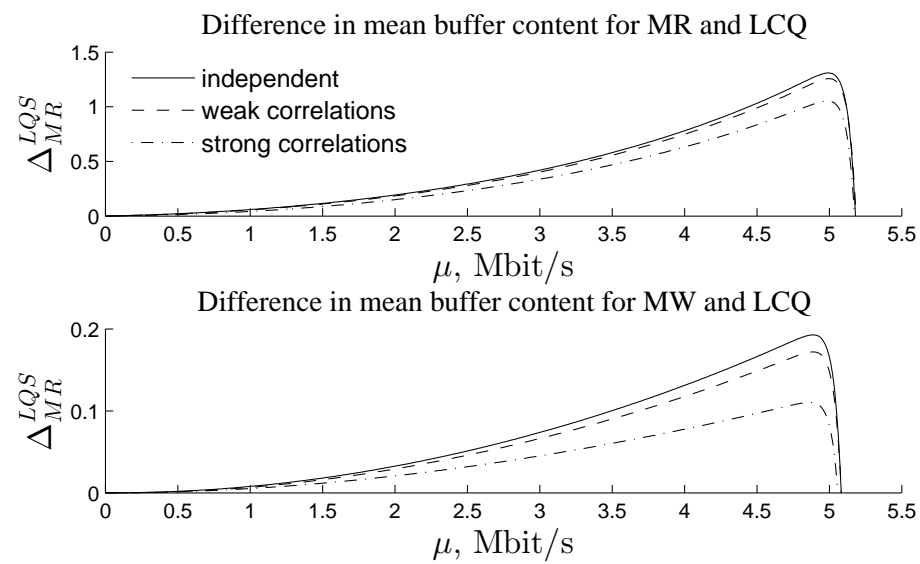

Difference in mean buffer content for GPS and DPS

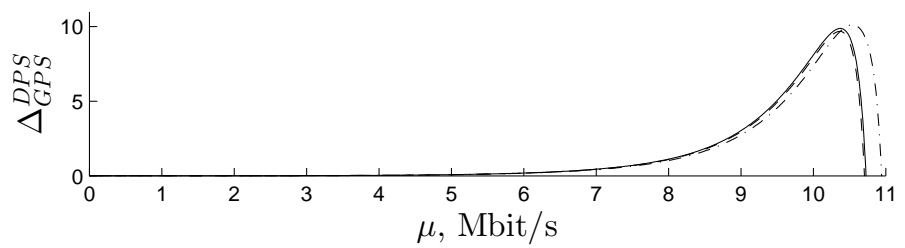

Figure 8: Difference in total mean buffer content in overload traffic for a system with $K=3$ mobile users under independent, weakly correlated and strongly correlated channels for MaxRate(MR), MaxWeight(MW), LCQ, DPS and GPS scheduling disciplines; the order of the expansion is $N=100$. 
and LCQ, and GPS and DPS,

$$
\begin{aligned}
\text { MaxRate and LCQ : } & \Delta_{\mathrm{MR}}^{\mathrm{LCQ}}=\mathrm{E}\left[Q^{\mathrm{LCQ}}\right]-\mathrm{E}\left[Q^{\mathrm{MR}}\right], \\
\text { MaxWeight and LCQ : } & \Delta_{\mathrm{MW}}^{\mathrm{LCQ}}=\mathrm{E}\left[Q^{\mathrm{LCQ}}\right]-\mathrm{E}\left[Q^{\mathrm{MW}}\right], \\
\text { GPS and DPS : } & \Delta_{\mathrm{GSP}}^{\mathrm{DSP}}=\mathrm{E}\left[Q^{\mathrm{DPS}}\right]-\mathrm{E}\left[Q^{\mathrm{GPS}}\right] .
\end{aligned}
$$

Note that a positive difference corresponds to a performance gain of the more opportunistic scheduler (MR, MW, GPS). For each comparison, we plot these differences for uncorrelated channels, for the Vine copula channel with rate matrix $\mathbf{R}_{2}$ (weak correlation), and for the Vine copula channel with rate matrix $\mathbf{R}_{3}$ (strong correlation). As previously noted, both MaxWeight and MaxRate outperform the LCQ scheduler, while GPS performs better than DPS, the performance gain being higher for less heavily loaded systems. The performance gain however clearly diminishes by the introduction of channel correlations, more correlation meaning less gain.

\section{Conclusions}

We considered a queueing model for assessing the performance of a downlink wireless MU transmission scenario, under varying channel conditions. The buffer behaviour of the wireless access point was modelled by a queueing system with multiple queues and a shared server. Accounting for time-correlation of the channel quality, the channels were modelled by an exogenous Markov process, each state of this Markov process corresponding to fixed (but not necessarily equal) channel qualities of the different channels.

As the state space of this system is very large and does not have additional structure which can be exploited (like product form, G/M/1-type or M/G/1-type), we focused on a numerical approximation approach which relies on series expansion techniques. We showed that this approach can calculate various performance measures fast in the light-traffic and the overload-traffic regimes.

We then adapted a discrete-time Markov model from literature for a single Rayleigh fading channel, first to an equivalent continuous-time Markov channel model, and then to a multi-channel Markov model. In the latter case, we relied on copulas (Clayton and Vine copulas) of the stationary distribution of the SNR of the different channels for the introduction of cross-channel correlation.

For the purpose of demonstration, several well-known scheduling disciplines were studied numerically. Our approximations for both independent and correlated channels were validated by simulation. It was shown that the approximation of the performance measures was not only computationally efficient, but also very accurate in the light-traffic and the overload-traffic regimes.

The proposed approach allows for several extensions. Most importantly, the Poisson assumption is not essential. The modelling assumptions can be extended to include arrival processes like interrupted Poisson processes and Markovian arrival processes. This can be achieved by introducing an arrival state for each 
arrival process which can easily be incorporated in the background process $M(t)$. A similar approach can be followed to relax the assumption that the transmission times are exponentially distributed. By introducing a service state for each queue and incorporating these states in the background process, the transmission times can follow a phase-type distribution. All these extensions however come with a computational cost as the state space of the background process will be considerably larger.

\section{References}

[1] J.W. Roberts. A survey on statistical bandwidth sharing. Computer Networks 45(3):319-332, 2004.

[2] S. Patil, and G. de Veciana. Measurement-based opportunistic scheduling for heterogenous wireless systems. IEEE Transactions on Communcations 57(9):2745-2753, 2009.

[3] X. Liu, E.K.P. Chong, and N.B. Shroff. Opportunistic transmission scheduling with resource-sharing constraints in wireless networks. IEEE Journal on Selected Areas in Communications 19(10):2053-2064, 2001.

[4] R. Knopp and P. Humblet. Information capacity and power control in single-cell multiuser communications. In: Proc. of IEEE ICC'95 “Gateway to Globalization”, vol. 1, pp. 331-334, June 1995.

[5] X. Liu, E.K.P. Chong, and N.B. Shroff. A framework for opportunistic scheduling in wireless networks. Computer Networks 41(4):451-474, 2003.

[6] X. Liu, E.K. Chong, and N.B. Shroff. Optimal opportunistic scheduling in wireless networks. In: Proc. of the 58th IEEE Vehicular Technology Conference, VTC 2003-Fall, vol. 3, pp. 1417-1421, 2003.

[7] W. Ajib and D. Haccoun. An overview of scheduling algorithms in MIMO-based fourth-generation wireless-systems. IEEE Network 19(5):43-48, 2005.

[8] A. Asadi and V. Mancuso. A survey on opportunistic scheduling in wireless communications. IEEE Communications Surveys \& Tutorials 15(4):1671-1688, 2013.

[9] J. Mietzner, R. Schober, L. Lampe, W.H. Gerstacker, and P.A. Hoeher. Multiple-antenna techniques for wireless communications-a comprehensive literature survey. IEEE Communications Surveys \& Tutorials 11(2):87-105, 2009.

[10] S. Shakkottai, T.S. Rappaport, and P.C. Karlsson. Cross-layer design for wireless networks. IEEE Communications Magazine 41(10):74-80, 2003.

[11] D. Gesbert, M. Kountouris, R.W. Heath, C.B. Chae, and T. Salzer,. Shifting the MIMO paradigm. IEEE Signal Processing Magazine 24(5):36-46, 2007.

[12] X. Lin, N.B. Shroff, and R. Srikant. A tutorial on cross-layer optimization in wireless networks. IEEE Journal on Selected areas in Communications 24(8):1452-1463, 2006.

[13] D. Gesbert and M. Slim-Alouini. How much feedback is multi-user diversity really worth? In: Proc. of the 2004 IEEE International Conference on Communications, vol. 1, pp. 234-238, 2004.

[14] P. Chaporkar, A. Proutiere, H. Asnani, and A. Karandikar. Scheduling with limited information in wireless systems. In: Proceedings of the tenth ACM international symposium on Mobile ad hoc networking and computing, pp. 75-84, 2009.

[15] B. Makki and T. Eriksson. Multiuser diversity in correlated Rayleigh-fading channels. EURASIP Journal on Wireless Communications and Networking 2012(1):1-9, 2012.

[16] M. Chiani, M.Z. Win, and A. Zanella. On the capacity of spatially correlated MIMO Rayleigh-fading channels. IEEE Transactions on Information Theory 49(10):2363-2371, 2003.

[17] B. Nosrat-Makouei, J. G. Andrews, and R. W. Heath Jr. MIMO interference alignment over correlated channels with imperfect CSI. IEEE Transactions on Signal Processing 59(6):2783-2794, 2011.

[18] L. Badia, A. Baiocchi, A. Todini, S. Merlin, S. Pupolin, A. Zanella, and M. Zorzi. On the impact of physical layer awareness 
on scheduling and resource allocation in broadband multicellular IEEE 802.16 systems. IEEE Wireless Communications $14(1): 36-43,2007$.

[19] B. Bellalta, A. Faridi, J. Barcelo, V. Daza, and M. Oliver. Queueing analysis in multiuser multi-packet transmission systems using spatial multiplexing. arXiv preprint arXiv:1207.3506, 2012.

[20] S. Shakkottai and A. L. Stolyar. Scheduling for multiple flows sharing a time-varying channel: The exponential rule. Translations of the American Mathematical Society-Series 2 207:185-202, 2002.

[21] W. Ajib and D. Haccoun. An overview of scheduling algorithms in MIMO-based fourth-generation wireless systems. IEEE Network 19(5):43-48, 2005.

[22] B. Sadiq, S.J. Baek, and G. De Veciana. Delay-optimal opportunistic scheduling and approximations: The log rule. IEEE/ACM Transactions on Networking 19(2):405-418, 2011.

[23] Y. Liu, S. Gruhl, and E.W. Knightly. WCFQ: an opportunistic wireless scheduler with statistical fairness bounds. IEEE Transactions on Wireless Communications 2(5):1017-1028, 2003.

[24] Z.H. Han and Y.H. Lee. Opportunistic scheduling with partial channel information in OFDMA/FDD systems. In: Proc. of the 60th IEEE Vehicular Technology Conference, VTC2004-Fall, vol. 1, pp. 511-514, 2004.

[25] O. Grøndalen, A. Zanella, K. Mahmood, M. Carpin, J. Rasool, O.N. Østerbø. Scheduling Policies in Time and Frequency Domains for LTE Downlink Channel: A Performance Comparison. IEEE Transactions on Vehicular Technology 66(4):3345-3360, 2017.

[26] H. Wei, L. Xiao, Y. Li, S. Zhou. Queue-aware Energy-efficient Scheduling and Power Allocation in Small-cell Networks with Interference. In: Proc of the IEEE Wireless Communications and Networking Conference (WCNC 2016), 2016.

[27] L. Lei, C. Lin, J. Cai, and X. Shen. Performance analysis of wireless opportunistic schedulers using stochastic Petri nets. IEEE Transactions on Wireless Communications 8(4):2076-2087, 2009.

[28] M.M. Rashid, M.J. Hossain, E.H. Hossain, and V.K. Bhargava. Opportunistic Spectrum Scheduling for Multiuser Cognitive Radio: A Queueing Analysis. IEEE Transactions on Wireless Communications 8(10):5259-5269, 2009.

[29] H.S. Wang and N. Moayeri. Finite-state Markov channel-a useful model for radio communication channels. IEEE Transactions on Vehicular Technology 44(1):163-171, 1995.

[30] P. Jacko, E. Morozov, L. Potakhina, I.M. Verloop. Maximal flowlevel stability of bestrate schedulers in heterogeneous wireless systems. Transactions on Emerging Telecommunication Technologies 28:e2930. doi: 10.1002/ett.2930, 2017.

[31] E. Altman, K.E. Avrachenkov, and R. Núñez-Queija. Perturbation analysis for denumerable Markov chains with applications to queueing models. Advances in Applied Probability 36(3):839-853, 2004.

[32] K. De Turck, E. De Cuypere, S. Wittevrongel, and D. Fiems. Algorithmic approach to series expansions around transient Markov chains with applications to paired queuing systems. In: Proc. of the 6th International Conference on Performance Evaluation Methodologies and Tools, pp. 38-44, 2012.

[33] E. De Cuypere, K. De Turck, and D. Fiems. A Maclaurin-series expansion approach to multiple paired queues. Operations Research Letters 42(3):203-207, 2014.

[34] K. De Turck and D. Fiems. A series expansion approach for finite-capacity processor sharing queues. In: Proc. of the 7th International Conference on Performance Evaluation Methodologies and Tools, pp. 118-125, 2013.

[35] J.B. Lasserre. A Formula for Singular Perturbations of Markov Chains. Journal of Applied Probability, 31(3):829-833, 1994.

[36] W.B. van den Hout. The power-series algorithm. PhD Thesis. University of Tilburg. 1996.

[37] B. Błaszczyszyn, T. Rolski, and V. Schmidt. Advances in Queueing: Theory, Methods and Open Problems, chapter: Light-traffic approximations in queues and related stochastic models. CRC Press, Boca Raton, Florida, 1995.

[38] I. Kovalenko. Rare events in queueing theory. A survey. Queueing systems 16(1):1-49, 1994.

[39] K. Avrachenkov and J.A. Filar. Analytic Perturbation Theory and Its Applications. SIAM, 2014. 
[40] E. Evdokimova, K. De Turck, S. Wittevrongel, and D. Fiems. Efficient Performance Evaluation of Wireless Networks with Varying Channel Conditions. In: Proc. of the 22nd International Conference on Analytical and Stochastic Modelling Techniques and Applications, pp. 59-72, Springer LNCS vol. 9081, 2015.

[41] E. Evdokimova, K. De Turck, S. Wittevrongel, and D. Fiems. An Analytical Performance Evaluation Tool for Wireless Access Points with Opportunistic Scheduling. In: Proc. of the 9th EAI International Conference on Performance Evaluation Methodologies and Tools, pp. 43-48, 2016.

[42] L. Tassiulas and A. Ephremides. Dynamic server allocation to parallel queues with randomly varying connectivity. IEEE Transactions on Information Theory 39(2):466-478, 1993.

[43] M. Andrews, K. Kumaran, K. Ramanan, A. Stolyar, R. Vijayakumar, and P. Whiting. Scheduling in a queueing system with asynchronously varying service rates. Probability in the Engineering and Informational Sciences 18(02):191-217, 2004.

[44] K.E. Avrachenkov and M. Haviv Perturbation of null spaces with application to the eigenvalue problem and generalized inverses. Linear Algebra and its Applications 369:1-25, 2003.

[45] P. Sadeghi, R.A. Kennedy, P. B. Rapajic, and R. Shams. Finite-State Markov Modeling of Fading Channels. IEEE Signal Processing Magazine 25(5):57-80, 2008.

[46] Q. Zhang and S. Kassam, Finite-state Markov model for Rayleigh fading channels. IEEE Transactions on Communications 47(11):1688-1692, 1999.

[47] K. Zheng, F. Liu, L. Lei, C. Lin, and Y. Jiang. Stochastic performance analysis of a wireless finite-state Markov channel. IEEE Transactions on Wireless Communications 2(12):1063-1072, 2013.

[48] A. Goldsmith. Wireless communications. Cambridge university press, 2005.

[49] R.B. Nelsen. An introduction to copulas. Springer Science \& Business Media, 2007.

[50] F. Durante, C. Sempe. Principles of Copula Theory. CRC Press, 2016.

[51] A. Panagiotelis, C. Czado, and H. Joe. Pair copula constructions for multivariate discrete data. Journal of the American Statistical Association 107(499):1063-1072, 2012.

[52] K. Aas, C. Czadob, A. Frigessic, H. Bakkend. Pair-copula constructions of multiple dependence Insurance: Mathematics and Economics 44:182-198, 2009.

[53] H.T. Banks, A. Broido, K. Gayvert, S. Hu, M. Joyner, and K. Link. Simulation and Modeling Related to Computational Science and Robotics Technology, chapter: Simulation Algorithms for Continuous Time Markov Chain Models. IOS Press, 2007.

[54] J. Aráuz, P. Krishnamurthy. A study of different partitioning schemes in first order Markovian models for Rayleigh fading channels. In: Proc of the 5th International Symposium on Wireless Personal Multimedia Communications, vol. 1, pp. $277-281,2002$.

[55] X. Ge, H. Jin, J. Zhu, J. Cheng, V.C.M. Leung. Exploiting Opportunistic Scheduling in Uplink Wiretap Networks, IEEE Transactions on Vehicular Technology 66(6):4886-4897, 2017.

[56] J.A. Hussein, S.S. Ikki, A. Boussakta, C.C. Tsimenidis. Performance Analysis of Opportunistic Scheduling in DualHop Multiuser Underlay Cognitive Network in the Presence of Cochannel Interference. IEEE Transactions on Vehicular Technology 65(10):8163-8176, 2016.

[57] H. Li, C. Huang, P. Zhang, S. Cui, J. Zhang. Distributed Opportunistic Scheduling for Energy Harvesting Based Wireless Networks: A Two-Stage Probing Approach. IEEE-ACM Transactions on Networking 24(3):1618-1631, 2016.

[58] K.N. Le, K.P. Dabke. Throughput analysis using opportunistic feedback, capture effect and MRT in Nakagami-m fading. Digital Signal Processing 63:177-189, 2017.

[59] D. Gesbert, H. Bölcskei, D.A. Gore, A.J. Paulraj. Outdoor MIMO Wireless Channels: Models and Performance Prediction. IEEE Transactions on Communications 50(12):1926-1934, 2002. 
[60] M. Elkashlan, T. Khattab, C. Leung, R. Schober. Statistics of general order selection in correlated Nakagami fading channels. IEEE Transactions on Communications 56(3):344-346, 2008. 